\title{
Study of CCD mosaic configurations for the ILMT: Astrometry and photometry of point sources in the absence of a TDI corrector
}

\author{
B. Vangeyte, J. Manfroid ${ }^{\star}$, and J. Surdej ${ }^{\star}$ \\ Institut d'Astrophysique et de Géophysique (IAGL), Université de Liège, Allée du 6 août, \\ 4000 Liège (Sart Tilman), Belgium
}

Received 21 February 2002 / Accepted 28 March 2002

\begin{abstract}
The image deformations induced by the time-delay integration (TDI) mode, used with a CCD camera at the prime focus of a Liquid Mirror Telescope (LMT), can be corrected for by a system of lenses, simultaneously with the optical aberrations. However, before adopting the design of a TDI corrector, we may wonder what is the importance of the TDI image deformations and how to possibly reduce these by choosing optimal mosaic configurations of CCDs, covering an equivalent field of view. In a first step, we have simulated the point spread function (PSF) deformation due to the TDI mode only, for different selected mosaic configurations. We have then estimated the importance of the astrometric and photometric errors induced by the TDI deformation. By simulating star fields with the same synthetic PSF and taking into account realistic sources of noise (cf. CCD readout noise, photon noise and sky background), we have derived the limiting magnitudes for photometry and astrometry for the case of a $4 \mathrm{~m} \mathrm{LMT}$.
\end{abstract}

Key words. methods: observationnal - techniques: miscellaneous - telescopes

\section{Introduction}

The surface of a liquid spinning with a constant angular velocity takes the shape of a paraboloid that can be used as the primary mirror of a telescope (Borra 1982). This idea was first suggested by E. Cappoci in 1850 (Mailly 1872). Liquid Mirror Telescopes (LMT) are zenith and may be used as survey telescopes. A consortium of several institutions is actively interested in developing a $4 \mathrm{~m}$ class LMT, named the International Liquid Mirror Telescope (ILMT), that could be installed at El Toco, in the North of Chile (Poels et al. 2001) or at Sinakas, in Crete.

The CCD at the prime focus of the ILMT will run in the TDI mode (also known as drift-scan mode or driftscanning). The electron transfer from column to column is made along a supposedly rectilinear trajectory, at the same speed as a star passing across the CCD centre, hereafter called the "central star". The integration time is thus equal to the time needed for the central star to go from one side of the CCD to the other side.

However, the trajectory of a star with declination $\delta$ in the CCD plane is not rectilinear and is represented by the following parametric equations (Stone et al. 1996), where

\footnotetext{
Send offprint requests to: B. Vangeyte, e-mail: vangeyte@astro.ulg.ac.be

* FNRS Research Director (Belgium).
}

$\xi$ and $\eta$ are respectively the positions along the East-West and the North-South axis, in a reference frame centred on the optical centre $o, \delta_{\mathrm{o}}$ is the declination of the telescope optical axis, $f$ is the focal length and $H$ is the hour angle:

$$
\begin{aligned}
& \xi=f \frac{\sin H \cos \delta}{\sin \delta_{\mathrm{o}} \sin \delta+\cos H \cos \delta_{\mathrm{o}} \cos \delta} \\
& \eta=f \frac{\cos H \sin \delta_{\mathrm{o}} \cos \delta-\cos \delta_{\mathrm{o}} \sin \delta}{\sin \delta_{\mathrm{o}} \sin \delta+\cos H \cos \delta_{\mathrm{o}} \cos \delta}
\end{aligned}
$$

This trajectory is conical. It results from the intersection of the focal plane with the cone described by the apparent motion of the star direction, neglecting the atmospheric refraction. From this nonrectilinear trajectory, image deformations result along the North-South axis, and also, because of the differential velocity, along the East-West axis.

Furthermore, there is an additional effect induced by the discrete shifting of the charges (Gibson \& Hickson 1992).

In the remainder, we study the importance of these effects by evaluating their influence on the trajectory (Sect. 3), on the PSF (Sect. 4) and on the astrometric and photometric precision (Sect. 5). All these calculations are made in the context of the ILMT project. Another application concerning an existing telescope working in 

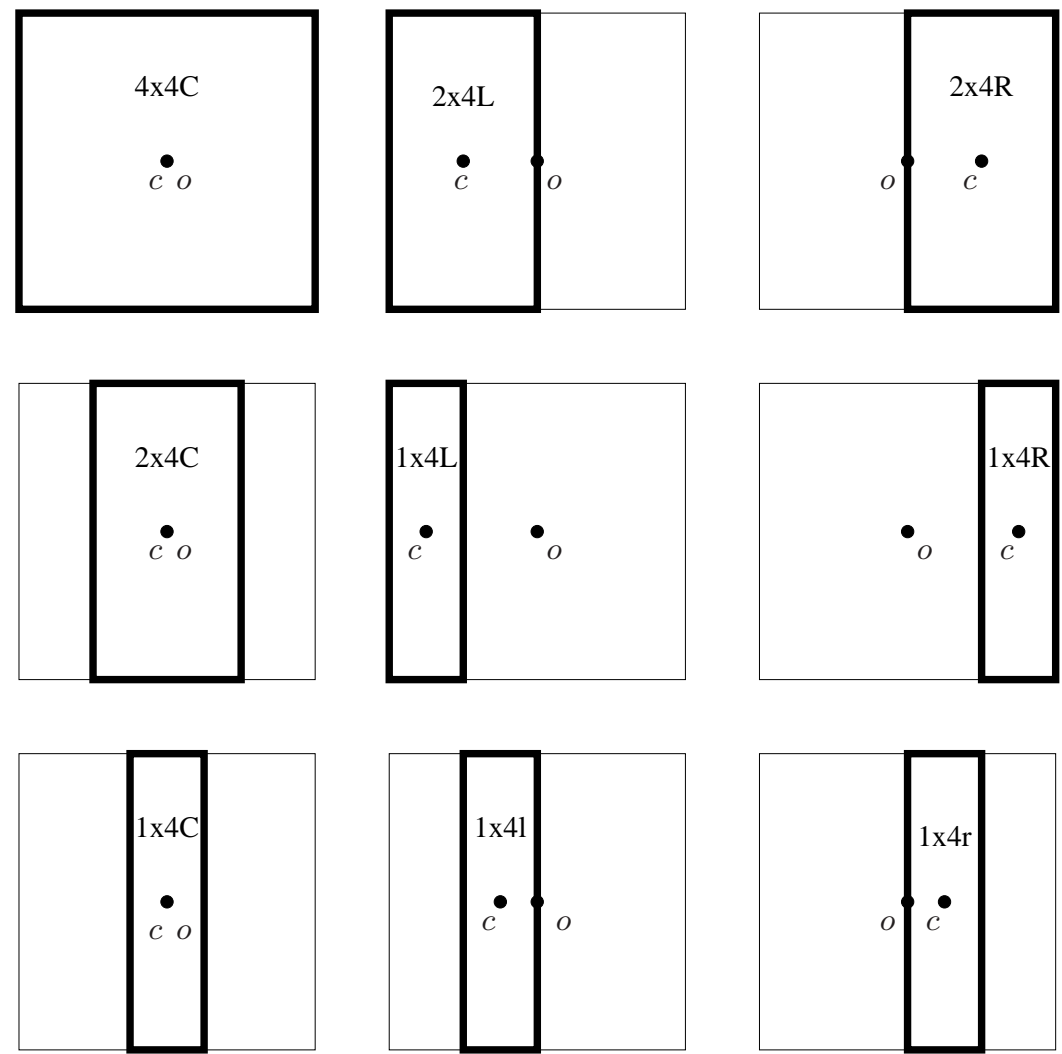

Fig. 1. Different single-CCD positions related to the same $4 \times 4$ equivalent field of view and used to calculate the resulting TDI image deformations.

the TDI mode is presented in Sect. 6. General conclusions form the last section.

\section{General description}

In order to evaluate the importance of TDI deformations on stellar images, we simulate the image integration of a star passing through the field of view. We assume that the effects of astigmatism, coma and distortion are perfectly corrected for by means of a conventional corrector.

This section presents our general assumptions, the adopted values for the most significant parameters in the case of the ILMT project, and the different mosaic configurations investigated. All these parameters are fixed in our study, but still remain open for the project.

\subsection{Telescope}

The telescope could possibly be installed at El Toco in Chile (latitude $\ell=-22^{\circ} 56^{\prime} 30^{\prime \prime}$ ). Focal length is $f=8 \mathrm{~m}$ and $\delta_{\mathrm{o}} \simeq \ell$, since the ILMT is a zenith telescope.

\subsection{Camera}

If $\delta_{c}$ is the declination of the centre $c$ of a single-CCD chip, not tilted, and composed of $m \times n$ pixels, $m$ being the number of pixels along the direction of the star trajectory, then the integration time, in seconds, is:

$$
T_{\mathrm{int}}=114.36 \frac{m}{4096} \frac{\text { scale }}{0.3867} \frac{\cos \left(-22^{\circ} 56^{\prime} 30^{\prime \prime}\right)}{\cos \delta_{\mathrm{c}}} .
$$

The camera will be made of a mosaic of CCDs whose pixel size is $p i x=15$ microns. The mosaic field size is $4096 \times 4096$ pixels. Then, the scale is:

scale $=\arctan \left(\frac{\text { pix }}{f}\right)=0.3867$ arcsec $/$ pixel.

\subsection{Single-CCD positions}

We shall investigate different mosaic configurations covering an equivalent field of view and composed of one or several CCDs. But first, we present the different singleCCD positions that we will study and use to derive what happens for the various mosaic configurations.

We start with a large CCD covering the whole mosaic field ( $m=4096$ pixels) and centred on the optical axis $\left(\delta_{\mathrm{c}}=\delta_{\mathrm{o}}\right)$. The resulting field of view side is $26.40 \mathrm{arcmin}$. In the remainder, we will refer to such a CCD as a $4 \times 4$ CCD.

Then, when composing the mosaic with several smaller CCDs, we shall talk of $2 \times 4,2 \times 2,1 \times 2$-CCDs, and so on. Figure 1 shows all single-CCD positions for which we calculate the resulting TDI image deformations. We shall refer to $2 \times 4 L, 2 \times 4 R$ and $2 \times 4 C$-CCDs, when they are 
respectively set on the left, on the right and at the centre of the mosaic. In the same way, we shall talk of $1 \times 4 L, 1 \times 4 l$, $1 \times 4 r, 1 \times 4 R$ and $1 \times 4 C$-CCDs, when they are respectively set at the extreme left, left, right, extreme right and at the centre of the mosaic.

\subsection{Trajectories}

The position of the star-trail in the $4 \times 4$ mosaic field is defined by a parameter which can take all values between 0.000 (star passing at the southern-bottom edge of the field) and 1.000 (star passing at the northern-top edge of the field). So we will refer to the trajectory of a star passing through the optical centre, i.e. a "central star", as to a "0.500 star-trail" or "0.500-trail" (see dashed curve in Fig. 2). In order to characterize a star passing close to the upper edge of the field, we shall speak of a "0.950-trail", and so on.

\subsection{Mosaic configurations}

By using the image deformation calculations made for the nine single-CCD positions presented in Sect. 2.3 and shown in Fig. 1, it is possible to deduce what happens for several realistic mosaic configurations, such as those illustrated in Fig. 3.

To evaluate the image deformations for configuration 2, we directly use the results from the $2 \times 4 L$ and $2 \times 4 R$-CCD positions. And for configuration 4 , we directly use the results from the $1 \times 4 L, 1 \times 4 l, 1 \times 4 r$ and $1 \times 4 R$-CCD positions.

Configurations 3 and 5 are the same as respectively configurations 2 and 4, except that the CCDs are slightly tilted by an angle $\psi$, i.e. their rows are aligned with the tangent to the star trajectory passing through the CCD centres. The results for these configurations can be deduced from the calculations of respectively the $2 \times 4 C$ and $1 \times 4 C$-CCD centred positions, assuming that the local radius of trail curvature from Eq. (1) does not vary across the field of view: this essentially constitutes a good approximation.

The worst images, i.e. in the sense of the TDI deformations, occur when the star passes at the northern or southern edge of the CCD. Then, for configurations 1 to 5 , we directly use the results from the single CCD positions, by simulating a 1.000-trail star (or a 0.000-trail one).

For configurations 6 to 10, we simulate a 0.750-trail star, because such a star corresponds to one passing at the extreme North of a $4 \times 2,2 \times 2$ or $1 \times 2$-CCD. While for configurations 11 to 15 , we use the results for a 0.625 -trail star in order to get information about the worst images. When doing so, we make the implicit assumption that the deformations do not vary if the CCD centre moves upwards or downwards through the mosaic field, i.e. if $\delta_{\mathrm{c}}$ slightly varies. This is a sound assumption.

\section{Star motion}

Let us calculate the star motion in the focal plane, i.e. the trajectory of the star centre. It depends on the latitude of

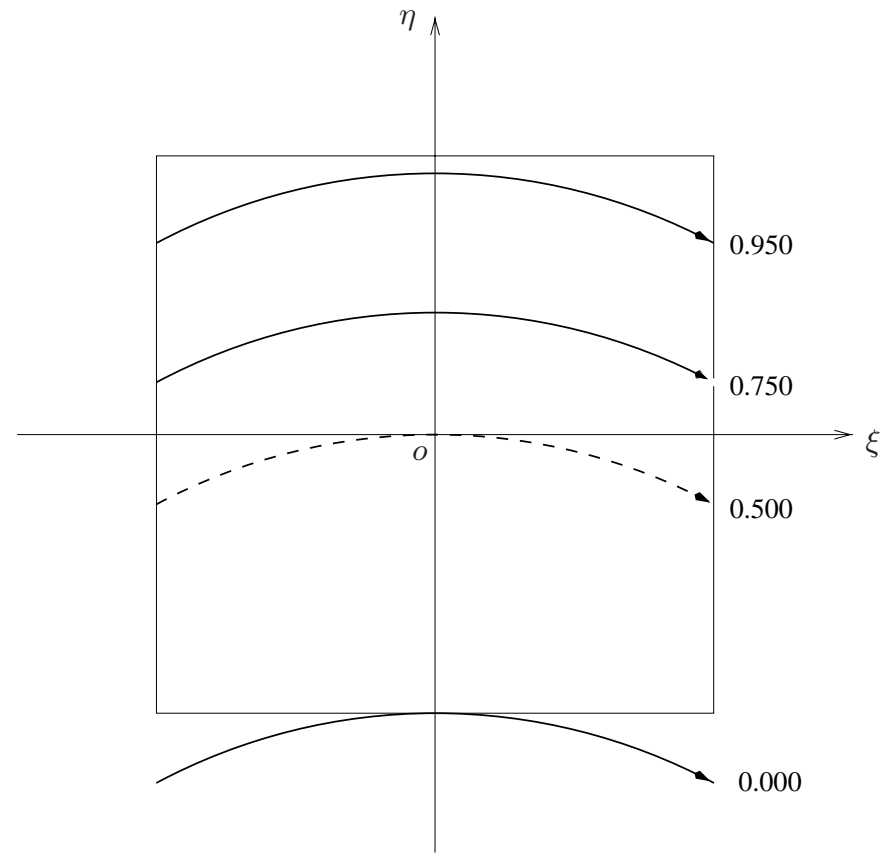

Fig. 2. Star trajectories passing across the ILMT field (southern hemisphere) and aligned along the direction of the star apparent motion ( $o \xi$ axis); the on axis points towards the North. The dashed curve illustrates the apparent trajectory of a star passing through the optical centre. Terminology: 0.950, $0.750,0.500$ and 0.000 -trails in the $4 \times 4 \mathrm{CCD}$ mosaic field.

the telescope, given its zenith use, and on the (relative) declination of the star, while the final image also depends on the size, position and orientation of the CCD.

\subsection{Star motion in the mosaic reference frame}

The mosaic of CCDs is placed in the focal plane whose centre $o$ coincides with the optical centre. By convention, the $o \xi$ axis points along the apparent stellar motion across the mosaic (West) and the on axis is defined by the direction of the North, all together defining the $o \xi \eta$ mosaic reference frame.

One CCD of the mosaic (see Fig. 4), made up of $m$ columns and $n$ rows, is fully defined by its centre $c$ coordinates in the $o \xi \eta$ reference frame and by the tilt angle $\psi$ between a column of pixels and the on axis. When the CCD is decentred, this tilt parameter allows the study of a CCD aligned with the star-trails.

The instantaneous position of the studied star $s$ is noted $s_{i}$, as reference to the hour angle $H_{i}$. The central star position $s^{\prime}$ having the same hour angle as $s_{i}$ is represented by $s_{i}^{\prime}$, while $\sigma$ is a virtual star whose centre follows a CCD row at the same speed as the charge transfer rate. We call it the "reference star" and it defines the "comoving reference frame", noted $\sigma x y$. Its axes are parallel to the CCD rows and columns and it is used to calculate the final image, as illustrated in Fig. 5.

The speed of the reference star (and of the comoving reference frame) is fixed by the speed of the central star: the TDI clock rate must be regulated so that $\sigma$ enters 
Configuration 1

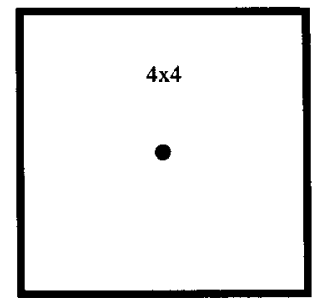

Configuration 6

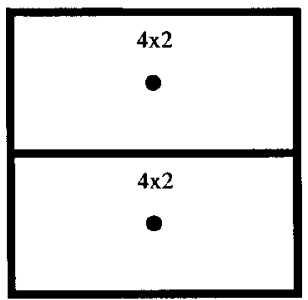

Configuration 11

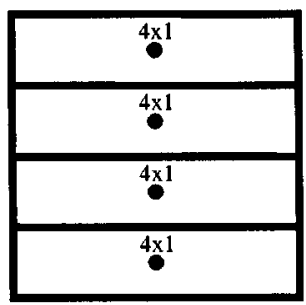

Configuration 2

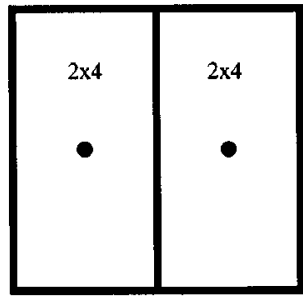

Configuration 7

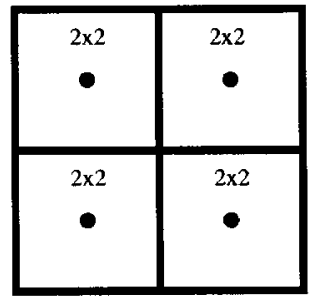

Configuration 12

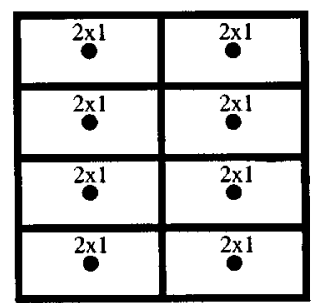

Configuration 3

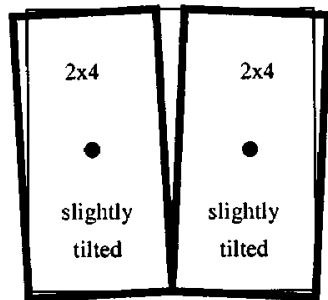

Configuration 8

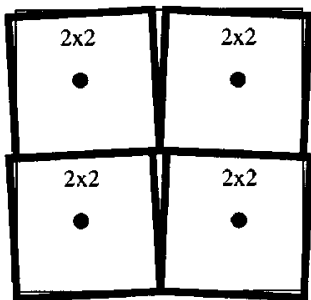

Configuration 13

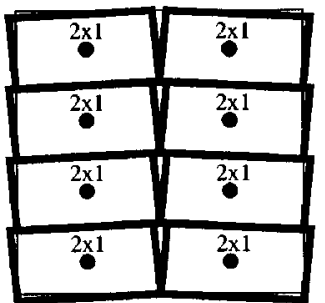

Configuration 4

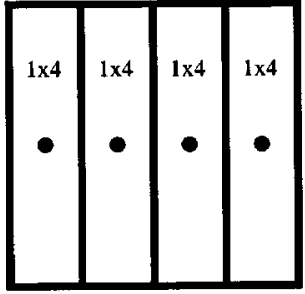

Configuration 9

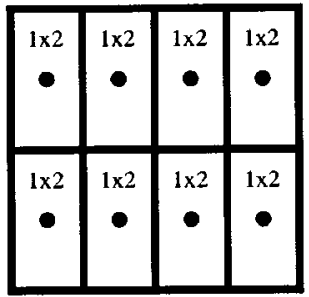

Configuration 14

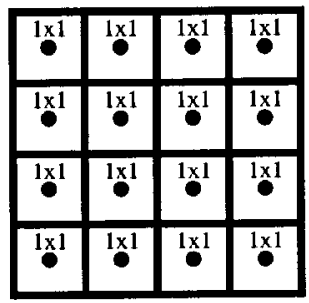

Configuration 5

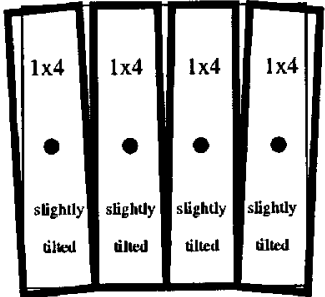

Configuration 10

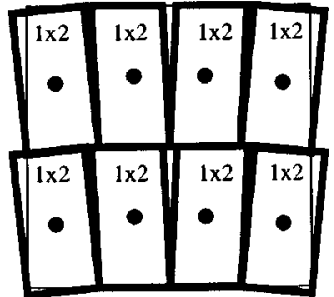

Configuration 15

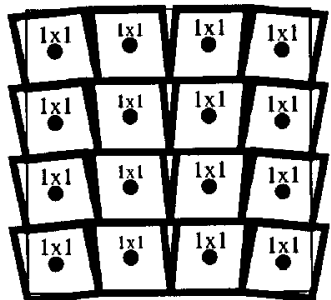

Fig. 3. Illustration of several possible mosaic configurations that have been investigated via the analysis of the 9 single-CCD positions shown in Fig. 1.

and goes out of the CCD simultaneously with $s^{\prime}$, passing through the CCD during the same span.

\subsection{Star trace in the comoving reference frame}

We construct the star image in the TDI mode by first calculating its centre's apparent motion relative to the $\sigma x y$ reference frame. The co-ordinates of the star centre in this frame describe the "trace" of the star.

When the TDI mode effects on the trajectory are perfectly corrected for, e.g. by a system of several lenses, see Hickson \& Richardson (1998), the studied star follows a rectilinear motion and the position of its centre always coincides with the reference star: $x=y=0$. The trace is then reduced to a point.

But when the camera is run in the drift-scan mode without any TDI correction, there are two different effects distorting the star motion and consequently, its trace. Figure 6 illustrates these two effects which are described hereafter.

\subsubsection{The curvature effect}

In the case of a central star (0.500-trail, see Fig. 6a), the clocking is optimal and there is only a relative motion according to the $\sigma y$ axis: $x=0$. Rigorously, $x \simeq 0$ because $\tan H \simeq H$; the star motion can be considered uniform for fields not wider than those considered for the ILMT $(\approx 30$ arcmin).

Due to the curvature of the trajectory, the position of the studied star centre is lower than that of the reference star centre at the time it enters the CCD $(y<0)$. When the star moves across the CCD field, its relative position then approaches $\sigma$, coinciding with it when the star passes at the meridian $(y=0)$ and then moves away from it again downwards as it goes out of the CCD $(y<0)$.

\subsubsection{The differential speed effect}

In the following, we assume a zenith telescope placed in the Southern hemisphere. Most of the effects discussed hereafter would be reversed in the northern hemisphere.

When the stellar trajectory is not central, e.g. to the North from the optical centre, in addition to the curvature effect, there is a speed variation effect: the star has a linear velocity larger than that of the central one. It enters the CCD after and exits before the latter one. The relative position is thus low on the left at the entry of the CCD $(x<0, y<0)$ and low on the right at the exit $(x>0$, $y<0)$. When the star-trail approaches the CCD top, the effect becomes worst, as seen by comparing Figs. 6b-6d. 


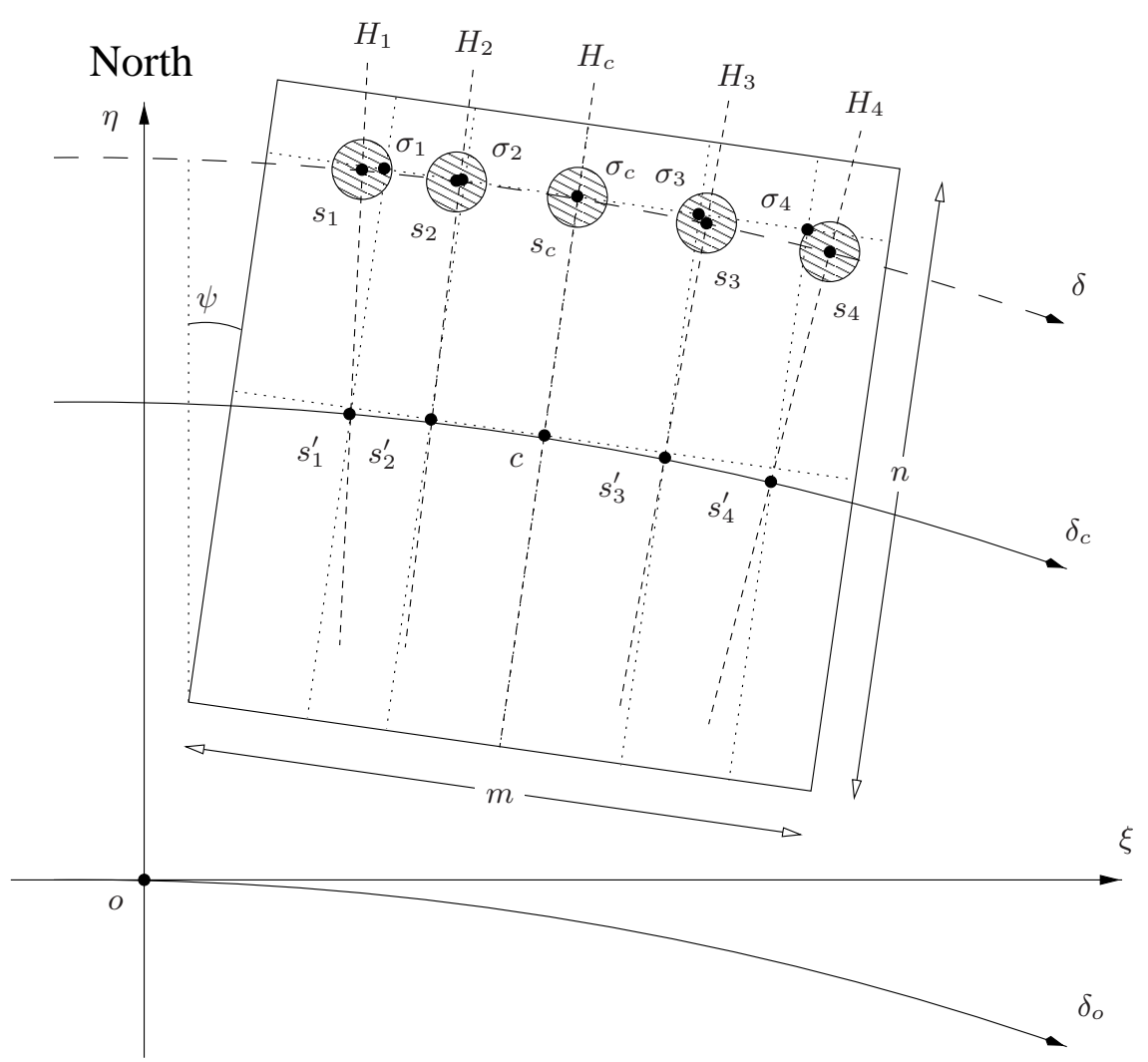

Fig. 4. Star motion in the mosaic reference frame, with one single CCD. $s_{i}$ is the studied star, $s_{i}^{\prime}$ is the central star and $\sigma_{i}$ a virtual reference star. The nearly vertical dashed lines represent celestian meridians, i.e. lines of constant hour angle. The light dotted lines refer to CCD rows and columns.

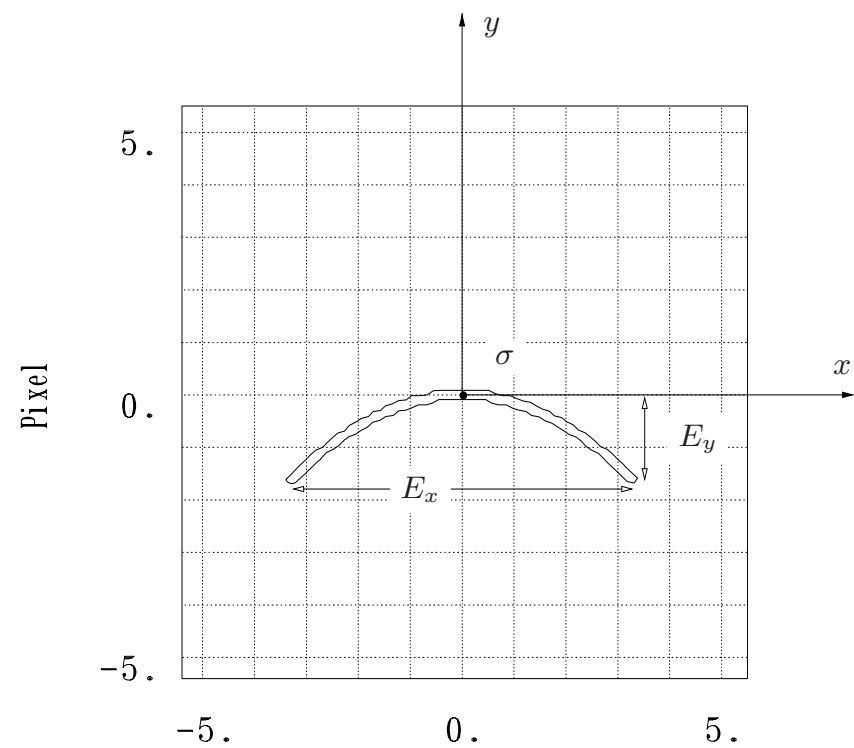

Fig. 5. Illustration of a trace produced by the TDI mode. Definition of the $\sigma x y$ comoving reference frame and of the $E_{x}$ and $E_{y}$ elongations.

To the South from the optical centre, the star has a linear velocity smaller than that of the central star. It enters the CCD before (relative position at the bottom right) and exits after (relative position at the bottom left). The final image for a 0.000 -trail star is quasi-identical to that for a 1.000-trail.
For a CCD centred on the optical centre axis, the differential speed effect is thus quasi-symmetrical for two trajectories that are equally distant from the central one.

To point out the difference between the image deformations of a 1.000 and a 0.000 -track, let us compare the traces for two star-trails with a $2 \times 4 L$-CCD. The traces in Figs. $6 \mathrm{e}$ and $6 \mathrm{f}$ show that the upper star enters before the reference star (left positioned) and that a lower star does it somewhat later (right positioned).

\subsection{Shifts from linearity}

The co-ordinates in the comoving reference frame $(x, y)$ represent the shifts from linearity. The change of coordinates between the mosaic and the comoving reference frame depends on the tilt angle $\psi$ :

$$
\left(\begin{array}{l}
x \\
y
\end{array}\right)=\left(\begin{array}{cc}
\cos \psi & -\sin \psi \\
\sin \psi & \cos \psi
\end{array}\right)\left(\begin{array}{c}
\xi-\xi_{\mathrm{CEN}} \\
\eta-\eta_{\mathrm{MER}}
\end{array}\right)
$$

where $\xi_{\mathrm{CEN}}$ is the central star position $\left(\delta=\delta_{\mathrm{c}}\right)$ having the same hour angle as the studied star and $\eta_{\mathrm{MER}}$ is the studied star position when it crosses the CCD centre meridian $\left(H=H_{\mathrm{c}}\right)$ (see Fig. 7$)$. These values are defined by the parametric Eq. (1):

$\xi_{\mathrm{CEN}}(H)=\xi\left(\delta_{\mathrm{c}}, H\right)$,

$\eta_{\mathrm{MER}}(\delta)=\eta\left(\delta, H_{\mathrm{c}}\right)$ 
For a non-tilted $\operatorname{CCD}(\psi=0)$, centred on the optical axis $\left(\delta_{\mathrm{c}}=\delta_{\mathrm{o}}, H_{\mathrm{c}}=H_{\mathrm{o}}=0\right)$, as illustrated in Fig. 7 , and by using a development in Mac Laurin series for $y$, the exact equations of motion reduce to:

$x(\delta, H)=-\frac{\xi_{\mathrm{CEN}}(H) \eta_{\mathrm{MER}}(\delta)}{f}\left(\frac{1+\tan \delta \tan \delta_{\mathrm{o}}}{\tan \delta+\cot \delta_{\mathrm{o}} \cos H}\right)$,

$y(\delta, H)=\frac{\xi^{2}(\delta, H)}{2 f \cot \delta}+\cdots$

By considering the nearly constancy over the whole field, of the curvature and of the term in parantheses in Eq. (4), Hickson \& Richardson (1998) deduced the following approximations (which are respectively their Eqs. (12) and (8)):

$x(\delta, H) \simeq x_{\mathrm{ap}}(\delta, H)=-\frac{\xi(\delta, H) \eta_{\mathrm{MER}}(\delta)}{f \cot \delta_{\mathrm{o}}}$,

$y(\delta, H) \simeq y_{\mathrm{ap}}(\delta, H)=\frac{\xi^{2}(\delta, H)}{2 f \cot \delta_{\mathrm{o}}}$

\subsection{Expected and approximated elongations}

We define the $E_{x}(\delta)$ and $E_{y}(\delta)$ elongations as being the longest distance between two points of the trace in the comoving reference frame, corresponding the hour angles $H_{\text {in }}$ and $H_{\text {out }}$ of a star entering and leaving out the CCD.

When the CCD is centred on the on axis $\left(H_{\mathrm{c}}=\right.$ $H_{\mathrm{o}}=0$, but not necessarily $\delta_{\mathrm{c}}=\delta_{\mathrm{o}}$ ), the final image is symmetrical with respect to the $\sigma y$ axis, so that $x\left(\delta, H_{\text {out }}\right)=-x\left(\delta, H_{\text {in }}\right)$ and $y\left(\delta, H_{\text {out }}\right)=y\left(\delta, H_{\text {in }}\right)$. For this case, we obtain:

$E_{x}(\delta)=2 x\left(\delta, H_{\text {out }}\right)$,

$E_{y}(\delta)=y\left(\delta, H_{\text {out }}\right)$.

Using the approximations of Hickson \& Richardson (1998), we finally obtain:

$E_{x}(\delta) \simeq E_{x_{\mathrm{ap}}}(\delta)=2 x_{\mathrm{ap}}\left(\delta, H_{\mathrm{out}}\right)$,

$E_{y}(\delta) \simeq E_{y_{\mathrm{ap}}}(\delta)=y_{\mathrm{ap}}\left(\delta, H_{\mathrm{out}}\right)$.

As expected, the elongations essentially depend on the $\delta$ declination. Let us consider the whole $4 \times 4$-mosaic field. Figure 8 illustrates the variations of the expected and approximated elongations against the latitude of the telescope, for a fixed chosen 0.000-track, while Fig. 9 shows it against the track, for a fixed chosen latitude $\left(\delta_{\mathrm{o}}=-22.9416^{\circ}\right)$.

The differential speed effect is much more important than the curvature effect and the $E_{x}$ elongation is maximum at the northern and southern edges. For two trails equally distant from the central trail, the approximated elongation values $\left(E_{x_{\text {ap }}}\right.$ and $\left.E_{y_{\text {ap }}}\right)$ are equal. This means that the TDI effects are quasi-symmetrical, but not strictly. Indeed, $E_{x}$ and $E_{y}$ are somewhat more important at the southern edge (0.000-track, i.e. the nearer to the nearest pole). It simply confirms that the $E_{x}$ and $E_{y}$ elongations very slightly decrease with declination.

\subsection{Maximum elongations}

Using Eqs. (5) and (7), we find:

$E_{x_{\mathrm{ap}}}(\delta)=-\frac{4 \eta_{\mathrm{MER}}(\delta)}{\xi\left(\delta, H_{\mathrm{out}}\right)} E_{y_{\mathrm{ap}}}(\delta)$.

Let us name $\delta_{\mathrm{S}}$ and $\delta_{\mathrm{N}}$, the declination of two stars following respectively the 0.000 and the 1.000 -tracks (cf. Fig. 7). At the southern edge of a $m \times n$-CCD, we have:

$\xi\left(\delta_{\mathrm{S}}, H_{\mathrm{out}}^{\mathrm{S}}\right)=m / 2$,

$$
\eta_{\mathrm{MER}}\left(\delta_{\mathrm{S}}\right)=-n / 2 .
$$

Then, we deduce a relation between the maximum elongations:

$$
E_{x_{\mathrm{ap}}}\left(\delta_{\mathrm{S}}\right)=\frac{4 n}{m} E_{y_{\mathrm{ap}}}\left(\delta_{\mathrm{S}}\right)
$$

$\Longrightarrow E_{x}\left(\delta_{\mathrm{S}}\right) \sim \frac{4 n}{m} E_{y}\left(\delta_{\mathrm{S}}\right)$.

This confirms the result obtained by Hickson \& Richardson (1998) for the case of a square CCD $(m=n)$, namely: $E_{x}\left(\delta_{\mathrm{S}}\right) \sim 4 E_{y}\left(\delta_{\mathrm{S}}\right)$ and also $E_{x}\left(\delta_{\mathrm{N}}\right) \sim-4 E_{y}\left(\delta_{\mathrm{N}}\right)$.

\subsection{Circular profile}

If we impose the TDI induced deformations to be the same in both directions, i.e. $E_{x}\left(\delta_{\mathrm{S}}\right) \simeq E_{y}\left(\delta_{\mathrm{S}}\right)$, then we must use a CCD with $m=4 n$. This implies that we expect configuration 11 (see Fig. 3) to be the best choice.

We will now verify this by analysing the point spread function resulting from the use of the TDI mode.

\section{Point spread function}

Let us now calculate the contribution of the TDI mode to the point spread function (PSF). A point source observed by the ILMT in the TDI mode, is affected by the distortion of the radiation wavefront produced by variations in the air refraction index (astronomical seeing), by the telescope response function (mirror and corrector lenses) and by the CCD operated in the TDI mode. The latter effect is composed of the pixel response function ( $\Pi$ ), the trace (see Sect. 3.2) and the discrete shifting response function $(\Lambda)$ (see e.g. Gibson \& Hickson 1992).

The pixel response function, noted $\Pi(x, y)$ and also shown in Fig. 10 along the $x$ axis, is inherent to all CCD operated in the classical mode and is not specific to the TDI mode. A contrario, the trace and the discrete shifting response function, noted $\Lambda(x)$ and shown in Fig. 10, are particular to the TDI mode. 


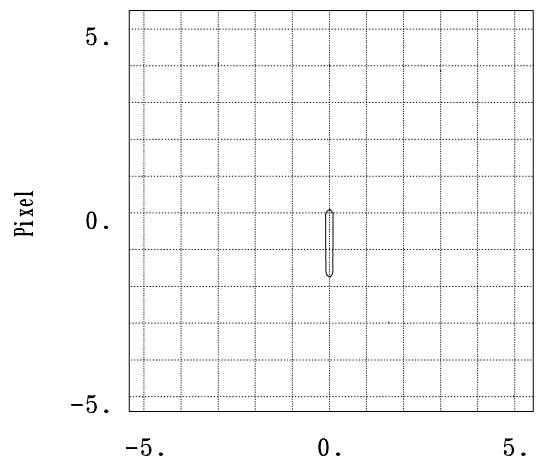

(a) $4 \times 4 C$ and 0.500 -trail.

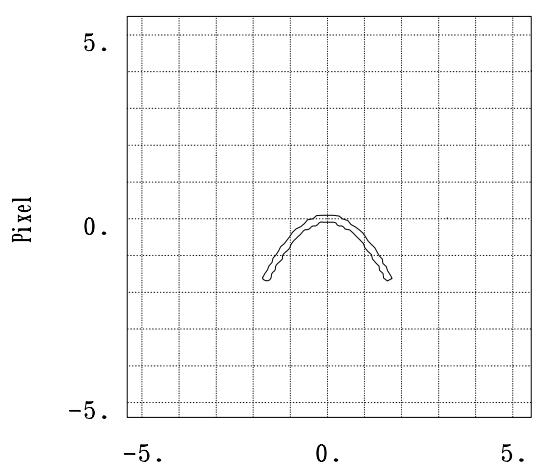

(c) $4 \times 4 C$ and 0.750 -trail.

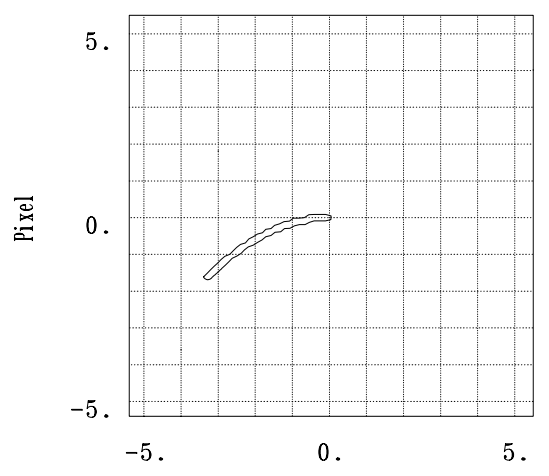

(e) $2 \times 4 L$ and 1.000-trail.

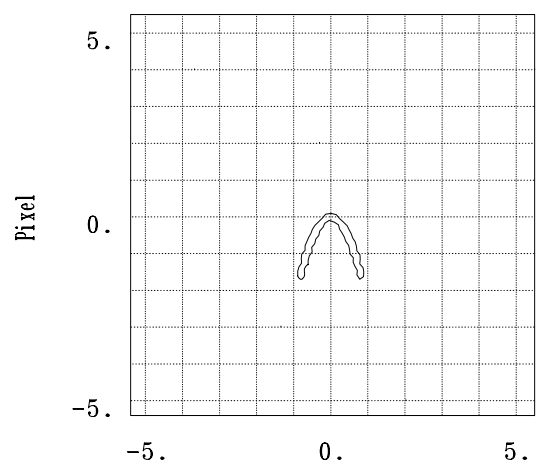

(b) $4 \times 4 C$ and 0.625 -trail.

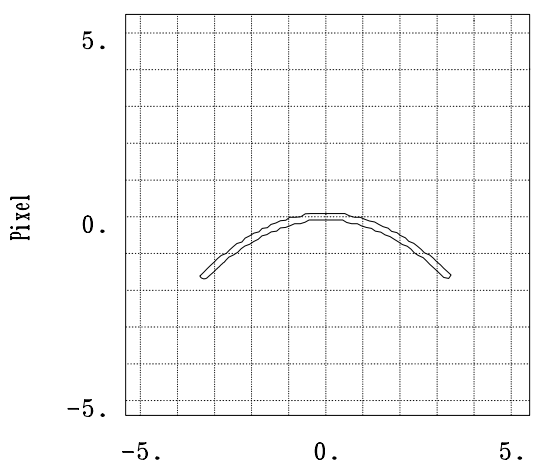

(d) $4 \times 4 C$ and 1.000-trail.

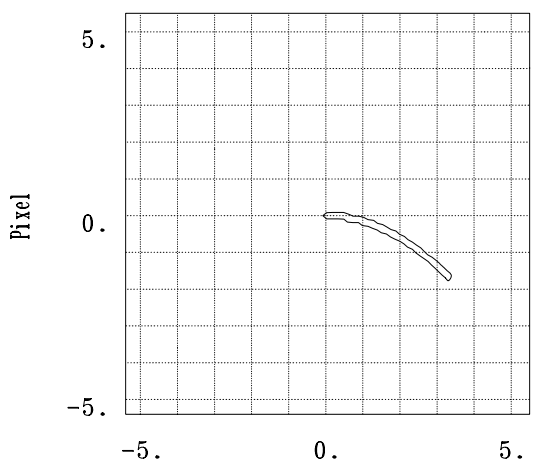

(f) $2 \times 4 L$ and 0.000 -trail.

Fig. 6. Star traces in the $\sigma x y$ comoving reference frame, for two different single-CCD positions and for several star-trail positions.

\subsection{The non-TDI contribution}

Let us first consider all the effects which are not specific to the TDI mode, which are namely the contributions of the seeing, the mirror, the corrector lenses and the $\Pi(x, y)$ pixel response function.

We assume that the image of a point source, produced through the instrument in the non-TDI mode, is invariant over the whole field of view and can be described by the so-called point spread function (PSF). In the remainder, for sake of simplicity, we assume that the PSF reduces to a Gaussian profile, characterized by a $F W H M$ in the range between 0.7 and 1.6 arcsec. This represents the combined effects due to the seeing, the parabolic mirror, the corrector lenses and the pixel response function. For simplicity, we will always refer to these four components as to the "seeing" which is assumed to be symmetrical in $x$ and $y$.

\subsection{The curvature and the differential speed effects}

As previously seen, two trajectory effects are outstanding. The curvature effect is responsible for the elongation of an image along the North-South direction ( $\sigma y$ axis), while 


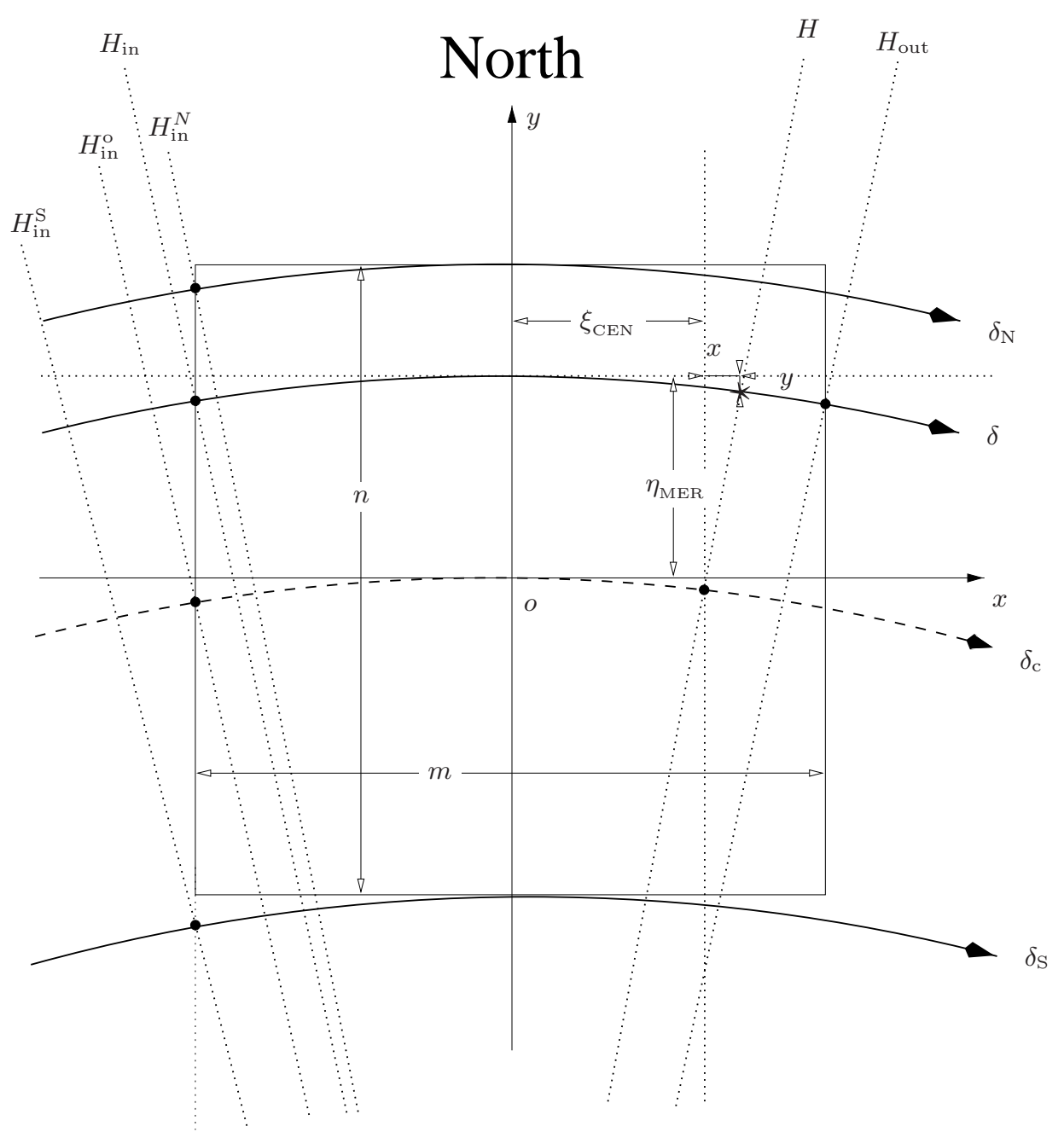

Fig. 7. Shifts $x$ and $y$ from linearity. Stars passing at the extreme North $\left(\delta_{\mathrm{N}}\right)$ and South $\left(\delta_{\mathrm{S}}\right)$ of the CCD.

the differential speed effect elongates this image along the East-West direction ( $\sigma x$ axis). The resulting trace in the comoving reference frame depends on the latitude, on the size, position and orientation of the CCD and on the star declination.

We thus have to convolve the Gaussian profile representing all the non-TDI effects, with the trace of the star in the comoving reference frame. This convolution can be calculated by a sum: after having calculated the relative motion in the comoving reference frame, we co-add all the individual images (refered to as "impacts" for simplicity) centred on the discrete $s_{i}$ points. Figure 11 shows the impact positions relative to the comoving reference frame.

\subsection{The discrete shifting effect}

The $\Lambda(x)$ function is caused by the discrete shifting of the charges whereas the incoming star centre continuously moves across the CCD. It mainly results into a smearing of the profile along the $x$ direction (Gibson \& Hickson 1992).

\subsection{Illustration of the TDI effects}

We now illustrate the relative importance of these effects by simulating the resulting PSF. Let us take, for example, a $4 \times 4 C$-CCD and a seeing of 0.7 arcsec. We just have seen that a star passing across the centre of the CCD (0.500trail) should only be deformed along the $\sigma y$ direction (see Fig. 12a).

Figures $12 \mathrm{~b}$ and $\mathrm{c}$ show how this profile gets deformed when the star passes higher in the field, respectively along the 0.750 and the 1.000 -trails.

\subsection{Comparison between the configurations}

In the present simulations, we have calculated all the profiles for four different seeings $(0.7,1.0,1.3$ and 1.6 arcsec) and for 41 different star trajectories (from 0.000 to 1.000).

For each of these 1476 numerically calculated profiles, we measure the $F W H M$, the peak, the position (in the comoving reference frame), the ellipticity and the ellipse tilt angle, as calculated by the IRAF routine IMEXAMINE, in order to compare the nine single-CCD positions presented in Fig. 1 and to consequently obtain a matter of choice between the mosaic configurations shown in Fig. 3. 


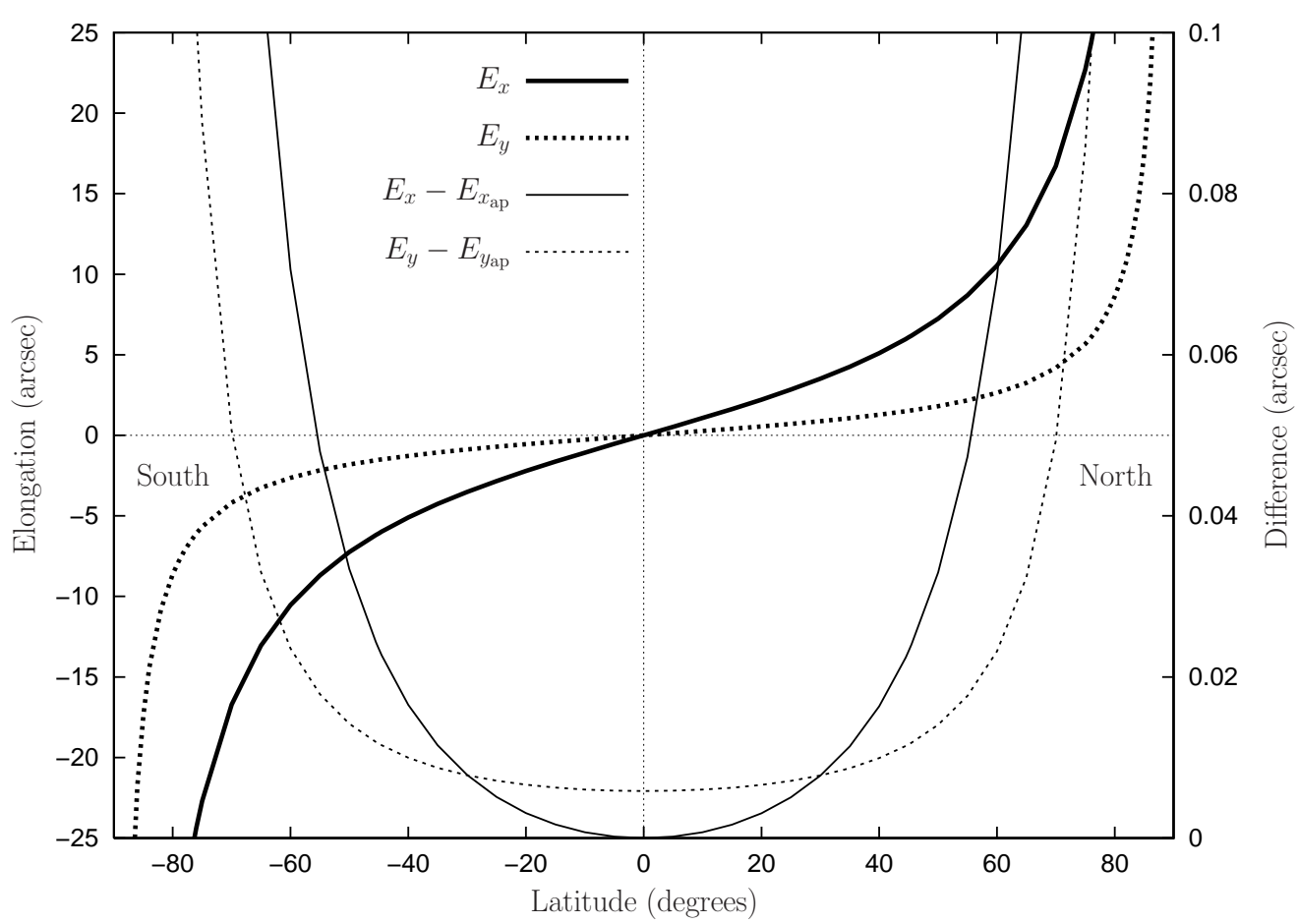

Fig. 8. Expected $(E)$ elongation and difference between $E$ and the approximated elongation ( $E_{\text {ap }}$ ), against the latitude, for a fixed 0.000 -track.

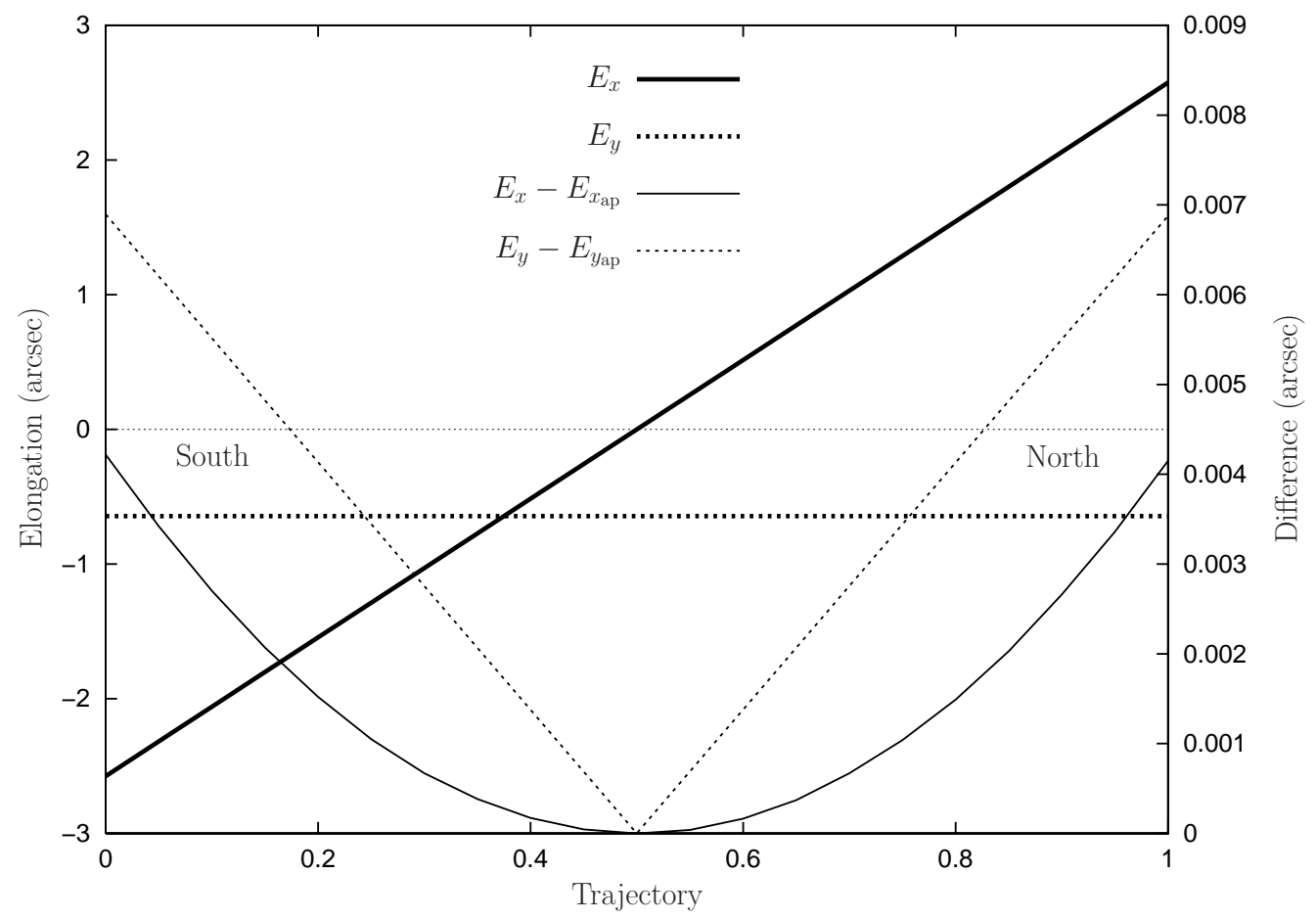

Fig. 9. Expected $(E)$ elongation and difference between $E$ and the approximated elongation $\left(E_{\text {ap }}\right)$, against the track co-ordinate, for a fixed latitude $\delta_{\mathrm{o}}=-22.9416^{\circ}$.

Figure 13 illustrates the influence of the TDI mode on the star profile, i.e. the ratio of the profile $F W H M$ to that of the Gaussian input of 1.0 arcsec (top graph), and the ratio of the star profile intensity peak to that of the Gaussian input (bottom graph), as a function of the star-trail position. Solid lines represent these ratios when taking the discrete shifting effect into account, while the dashed lines do not include this effect.

Figure 14 illustrates the relative influence of the curvature versus the differential speed effects, measuring the profile ellipticity (top graph) and its orientation (ellipse angle, bottom graph). This confirms that the elongation 


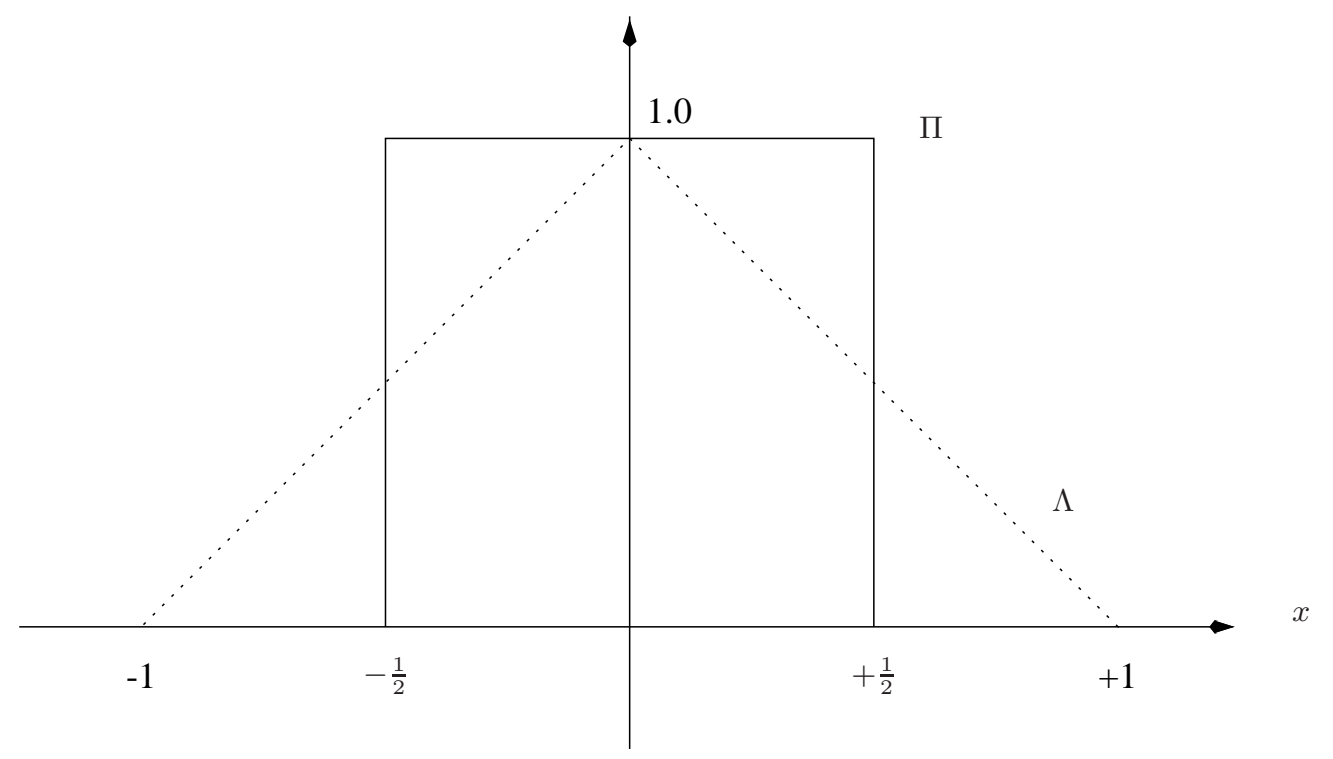

Fig. 10. The $\Pi(x, y)$ pixel response function and the $\Lambda(x)$ discrete shifting response function, along the $x$ axis, for which units correspond to pixels.
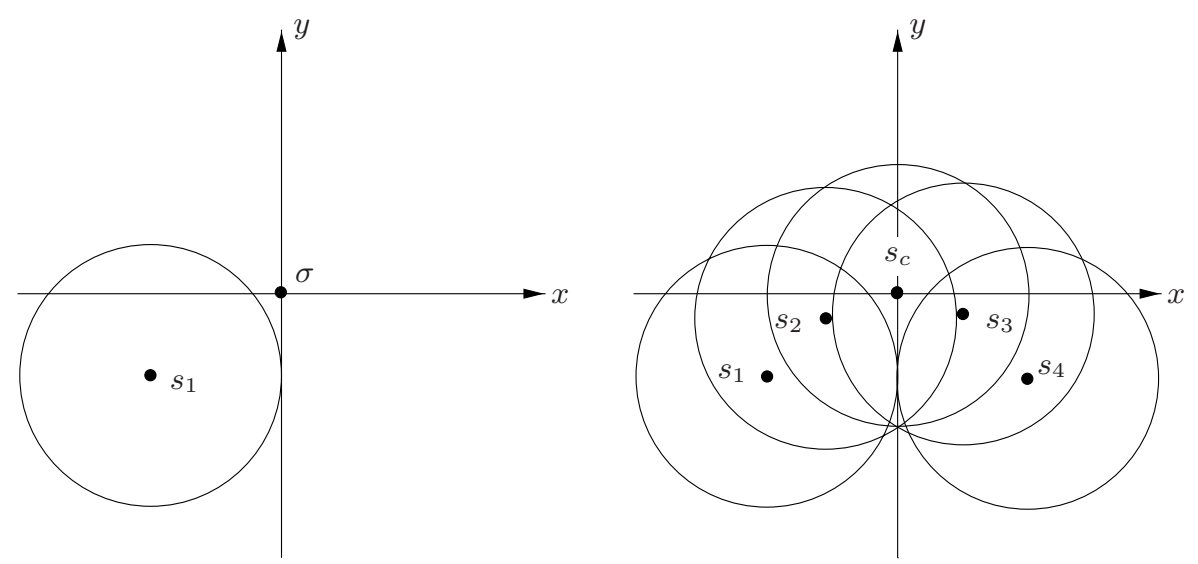

Fig. 11. Star motion in the comoving reference frame $\sigma x y$. One impact (on the left) and five impacts (on the right).

of a 0.500 -trail is essentially oriented along the $\sigma y$ axis (angle $\sim 90^{\circ}$ ), highlighting the dominance of the curvature effect.

Note that for the large $4 \times 4$ considered field, ellipticity is minimum for the 0.375 and 0.625 -tracks, confirming how to obtain a circular PSF, e.g. with configuration 11, which, as mentioned in Sect. 3.6, gives approximately equal $E_{x}$ and $E_{y}$. When taking the discrete shifting into acount, these minima are displaced respectively to the 0.425 and 0.575 positions. But this does not change the conclusion of using configuration 11, because the track range (from 0.375 to 0.625 positions) for which the ellipticity remains reasonably small corresponds to the height (North-South) of the $1 \times 4$-CCDs to be used in this configuration.

\subsection{Cutting out of the mosaic}

In order to make a good choice between the proposed configurations, we can basically follow two approaches of cutting out the mosaic.

\subsubsection{North-South cutting out}

For the given latitude and seeing, we deduce that the curvature effect ( 0.500 track) does not affect the $F W H M$ by more than $15.5 \%$. Nevertheless, the differential speed effect enlarges the PSF more when the star passes closer to the top or bottom edges (rising up to $58.6 \%$ for a 0.000 track, for a large $4 \times 4$-CCD). Figure 13 reveals that this kind of reasoning also nicely applies to the behavior of the intensity peak ratio.

From this, we may conclude that it is better to divide the CCD into a mosaic of narrower CCDs along the North-South direction, in order to avoid a too big difference between the charge transfer clocking and the real star speed. From the analysis of the shape of the curves, we clearly see that dividing by two or four (2048 or 1024 pixels North-South in this case) is reasonable. But more than four would be unproductive, because of the flatness of the curves around the central track. 

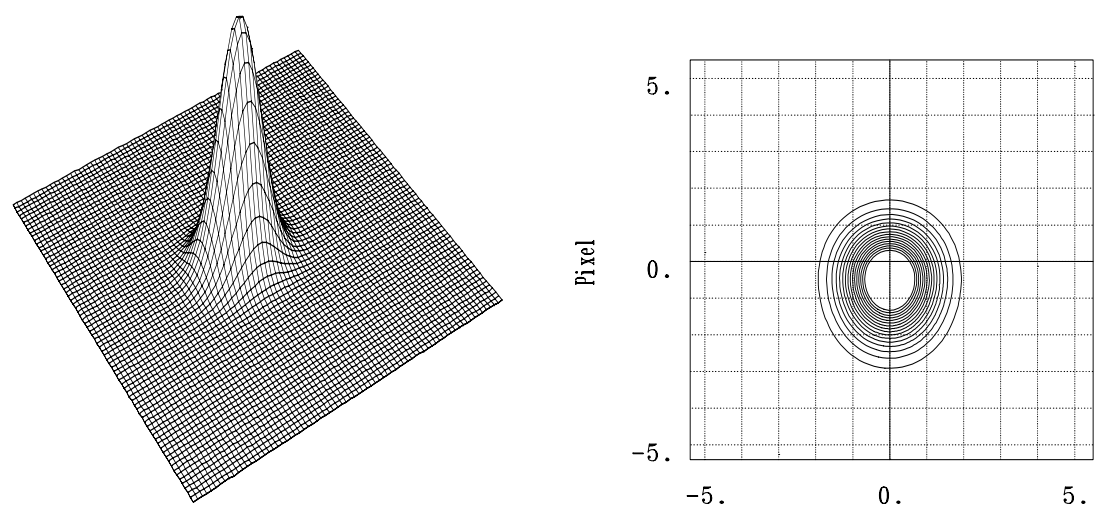

(a) 0.500 -trail.
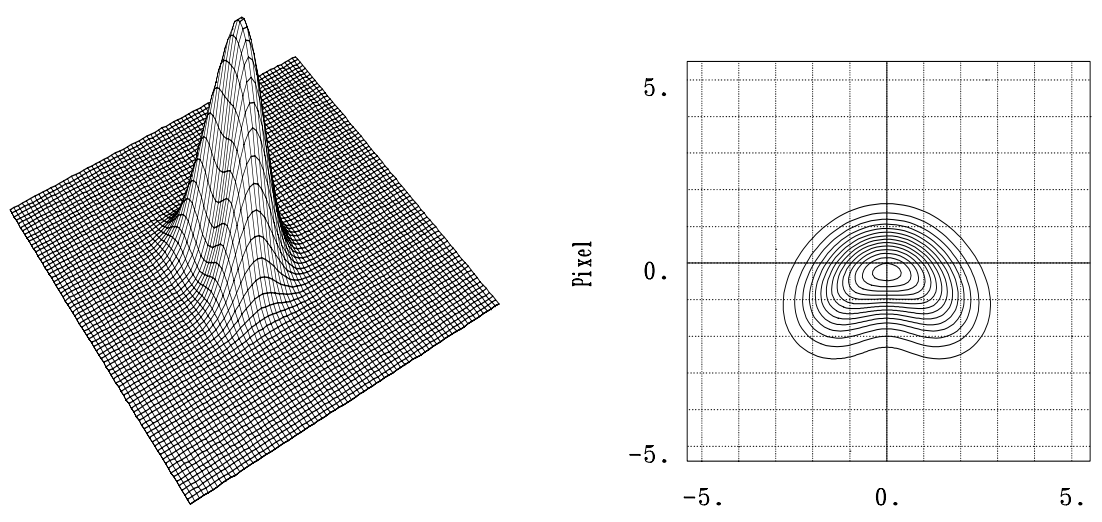

(b) 0.750 -trail.
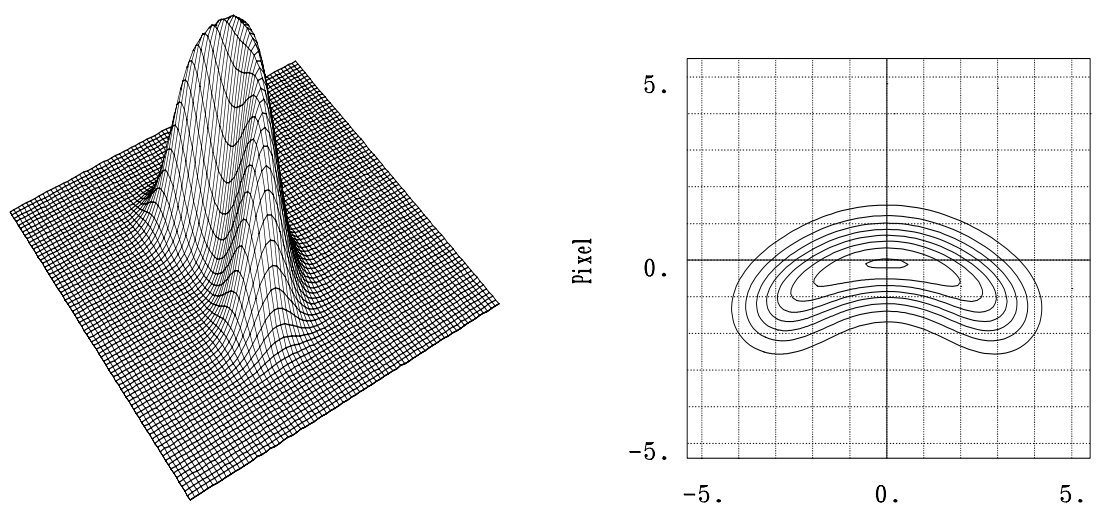

(c) 1.000-trail.

Fig. 12. The PSF deformed by the TDI mode, i.e. the curvature effect, the differential speed effect and the discrete shifting effect, in the case of a $4 \times 4 C$-CCD, for a seeing of 0.7 arcsec. Major ticks are separated by 1 pixel $(\simeq 0.4 \operatorname{arcsec})$.

Note that the discrete shifting clearly affects the $F W H M$ and is relatively more important for a central star (see dashed versus solid lines in Fig. 13).

\subsubsection{East-West CCD cutting out}

Another way to reduce the bad impact of the TDI on the $F W H M$, is to use a narrower CCD along the East-West direction: it is clear that going from a $4 \times 4 C$ to a $2 \times 4 C$ or to a $1 \times 4 C$ significantly improves the $F W H M$ ratio. But when doing this, it also shortens the integration time. Thus, we will have to investigate how the TDI effects act on the astrometric and photometric uncertainties, which are, of course, dependent on the corresponding integration times. 


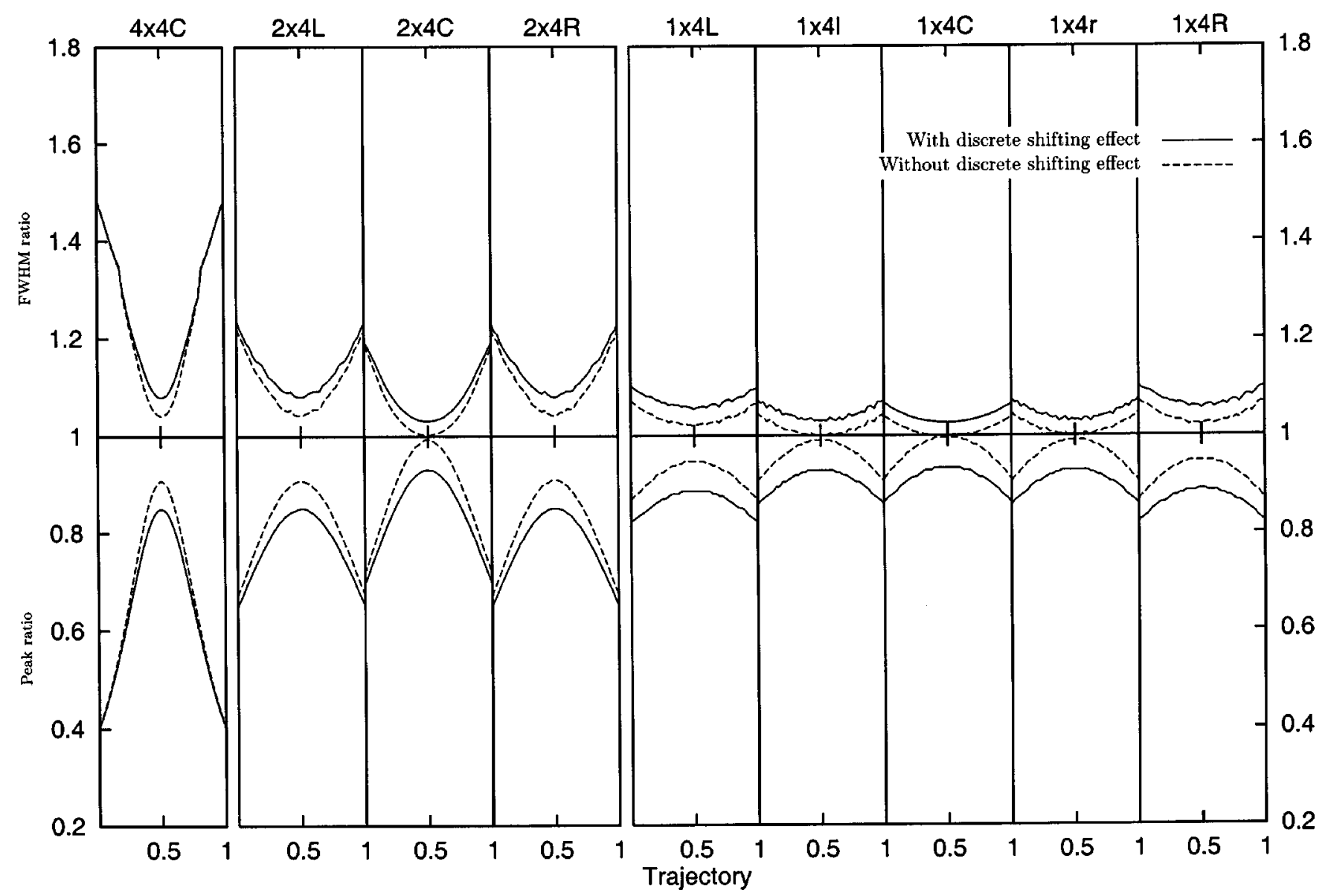

Fig. 13. Star intensity peak to Gaussian peak ratio (bottom) and star profile $F W H M$ to Gaussian $F W H M$ ratio (top), as a function of the star-trail position, for the various CCD locations presented in Fig. 1, and for a seeing of 1.0 arcsec.

All these considerations must now be confronted to the analysis of the TDI influence on the astrometric and photometric accuracies.

\section{Astrometric and photometric uncertainties}

In this section, we report the astrometric and photometric uncertainties resulting from the TDI mode.

\subsection{Star frame simulations}

For statistical purposes, we simulate a large number of frames of stars with known positions and magnitudes, being all characterized by the same PSF, i.e. having the same declination (trail), for the same single CCD-configuration and for the same seeing. More precisely, for each simulation, we construct a working frame of e.g. $1024 \times 1024$ pixels, filled with 2500 stars well aligned to avoid blended stars, as seen in Fig. 15. This case corresponds to a $4 \times 4 C$ CCD, a seeing of 0.7 arcsec and a 0.950-trail star. Each star has its own specific magnitude in the range between 20 and 25.

We have chosen a sky background of $65 \mathrm{e}^{-} / \mathrm{sec} / \operatorname{arcsec}$ corresponding to a moonless $V$ sky brightness of $21.8 \mathrm{mag} /$ arcsec. The adopted atmospheric extinction is typical of Chilean observations. We add photon noise and take 8.5 electrons for the CCD readout noise. The CCD quantum efficiency is taken to be $80 \%$. The adopted CCD gain is $1 \mathrm{adu} / \mathrm{e}^{-}$.

\subsection{Error measurements}

For measurements, we use the IRAF aperture photometry routine PHOT and the IRAF PSF-fitting routine ALLSTAR. In order to make the comparison for a large number of simulations in exactly the same conditions, we keep all the parameters fixed for each configuration, each seeing, each trajectory, etc. Of course, we could possibly find better values in some cases but our purpose here is to make coherent and automatic comparison between all the simulations.

Note that for the PSF-fitting, we use the analytical calculated synthetic PSF (as explained in Sect. 4.2), thus well adapted for the given star-trail position: this will not be feasible in real conditions and it will be necessary to compute the PSF from non blended bright stars having relatively the same trail position.

Figure 16 shows the results of one simulation, i.e. the error measurements corresponding to a star passing through a $4 \times 4$-CCD, for a 1.000 -trail and a seeing of 1.0 arcsec. We have represented, as a function of the stellar magnitude, the differences between the exact input 


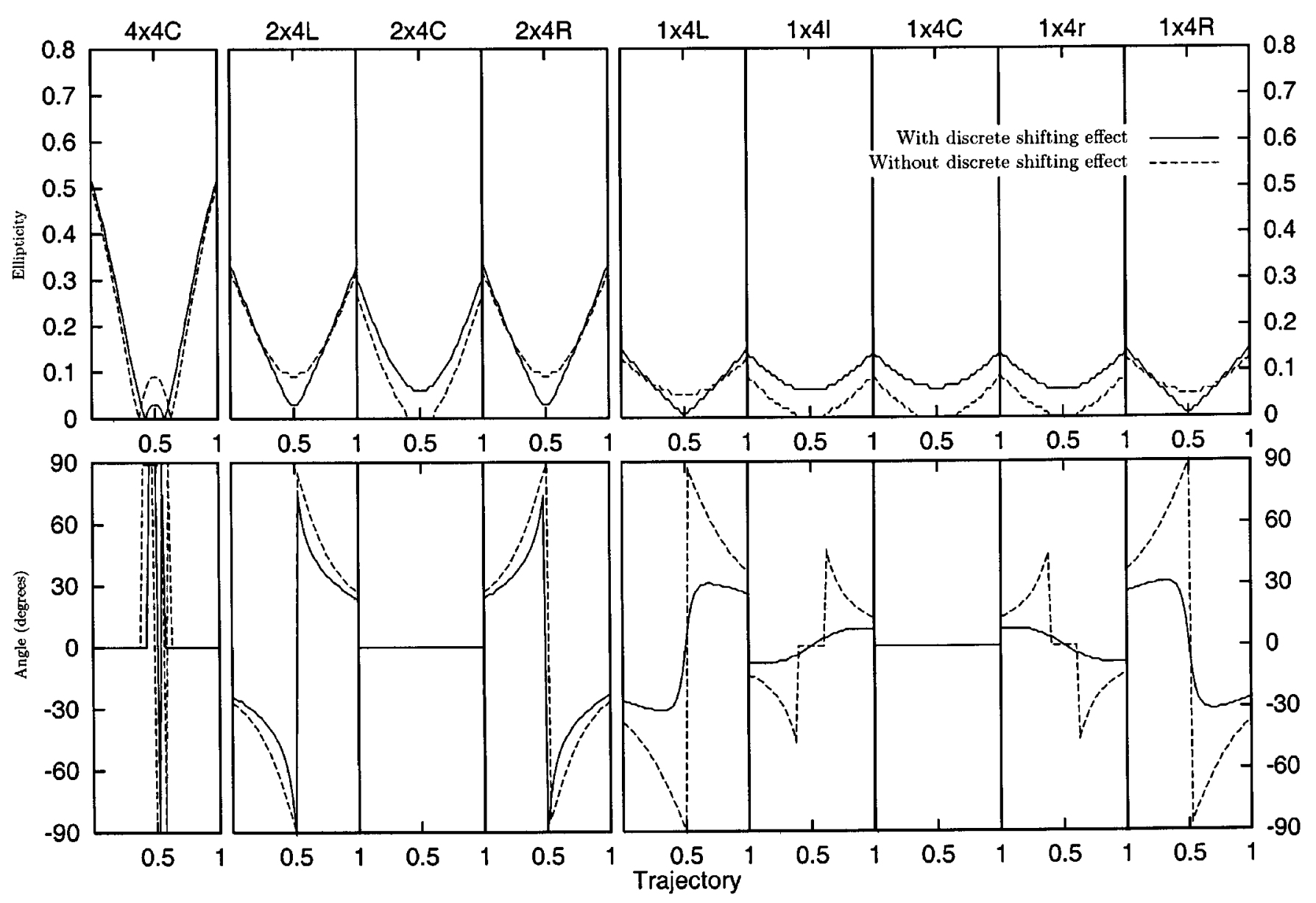

Fig. 14. Ellipticity (top) and ellipse tilt angle (bottom), as a function of the star-trail position, for the various CCD locations presented in Fig. 1, and for a seeing of 1.0 arcsec.

quantities (position and magnitude) and their measurement with the IRAF routines applied to the simulated data: the astrometric errors in Fig. 16a are obtained by quadratically summing up the errors along the $\sigma x$ and $\sigma y$ axes. Similarly, the photometric errors are presented in Fig. 16b.

\subsection{Error curves}

For each graph (see Fig. 16), we summarize the error behavior against the stellar magnitude by fitting a curve at $1 \sigma$, such that $68.27 \%$ of the points lie under it. We use a sliding median for calculating it.

In order to compare the results for the different CCD mosaic configurations, we use the sliding median curves from each case, like the ones in Fig. 16, as illustrated in Fig. 17a for the astrometry and in Fig. 17b for the photometry.

\subsection{Limiting magnitudes}

The limiting magnitude is determined when the sliding median curve cuts a chosen upper error limit. For the astrometry and for the various configurations, we choose a limiting magnitude corresponding to an error of 0.1 pixel, that is to say $\approx 0.04$ arcsec (horizontal straight line in Figs. 16a and 17a), because this is typically the intrinsic precision limit of the atmosphere (Viateau et al. 1998).

For the photometry, we choose a limiting magnitude corresponding to an error of 0.2 mag. Because such an error corresponds to a flux ratio of $20 \%$, it roughly corresponds to stars with a signal to noise ratio of $S / N=5$ (horizontal straight line in Figs. 16b and 17b).

For statistical purposes, we make $N$ simulations of the same case. Then we derive the $N$ corresponding error curves and the $N$ limiting magnitudes. The median of these values is noted $m_{\lim [50]}$. In order to obtain an estimated value of the accuracy, we also calculate $m_{\lim [16]}$ (resp. $\left.m_{\lim [84]}\right)$, as the value for which $16 \%$ (resp. 84\%) of the magnitudes are brighter in the whole sorted list of limiting magnitudes. This gives an error bar inside which lie $68.27 \%$ (corresponding to $1 \sigma$ ) of the points.

\subsection{Comparison between the configurations}

In the present simulations, we have constructed star frames for six configurations of the nine previously defined ones $(2 \times 4 R, 1 \times 4 r$ and $1 \times 4 R$ being in principle the 

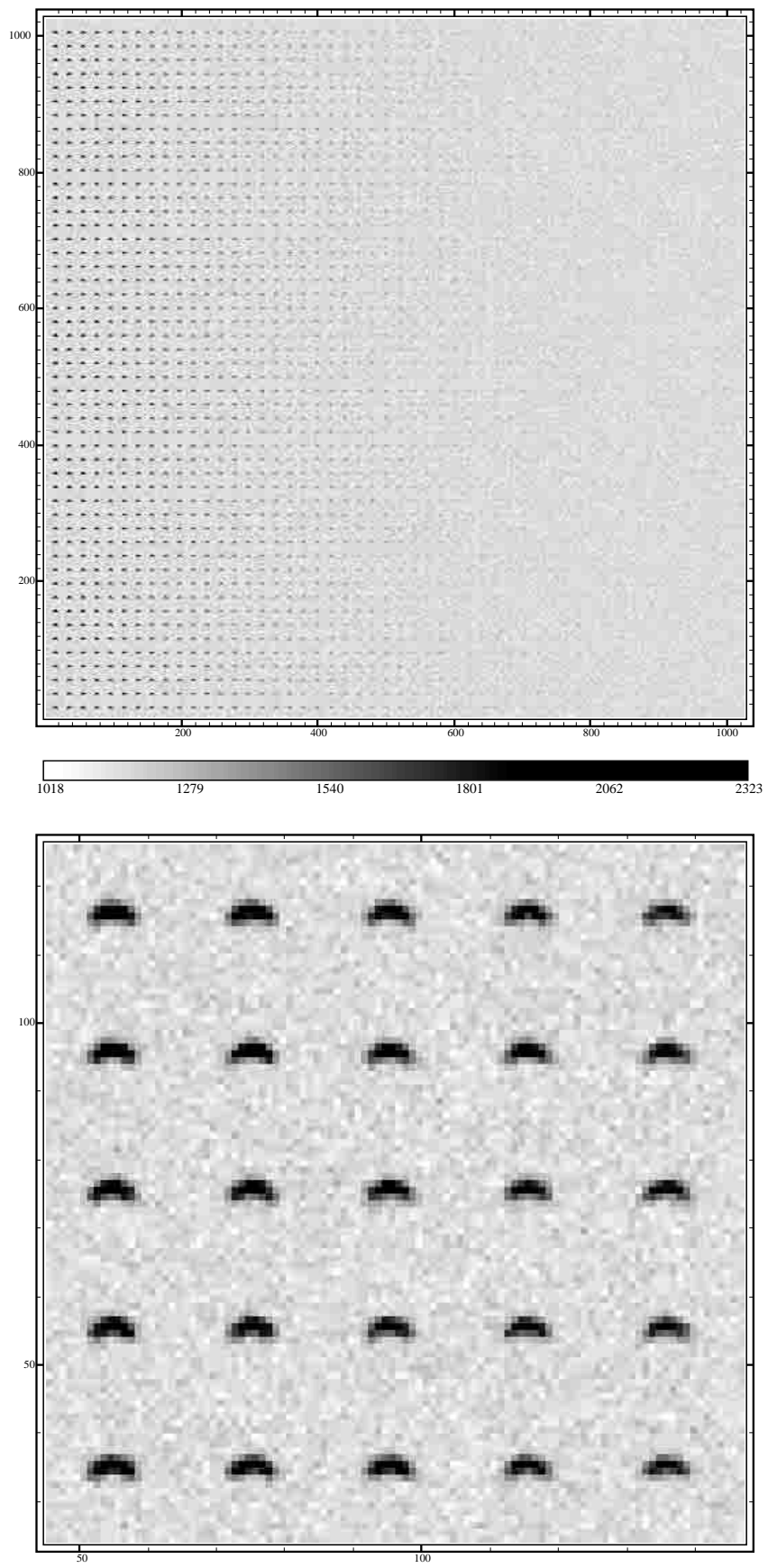

Fig. 15. Simulated frame for statistical purposes (with a zoomed part of it shown in the bottom graph), filled with 2500 stars having a PSF corresponding to a $4 \times 4 C$-CCD, a seeing of 0.7 arcsec and a 0.950 -trail star.

symetrical cases of, respectively, $2 \times 4 L, 1 \times 4 l$ and $1 \times 4 L$ ), for four different seeings $(0.7,1.0,1.3,1.6$ arcsec $)$, using the PSF-fitting and aperture photometry methods, in order to measure the $o \xi$-position, the $o \eta$-position, the position and the photometry, for four different star trajectories $(0.500$, $0.625,0.750$ and 1.000-tracks).

We $\operatorname{did} N=14$ times those simulations where the TDI was perfectly corrected for (i.e. 2688 error graphs, these

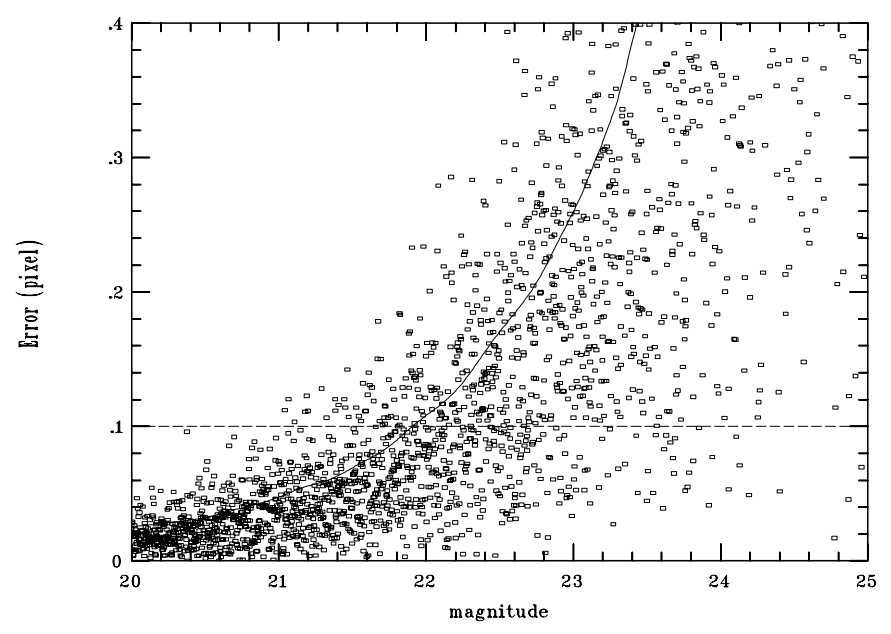

(a) Astrometric errors.

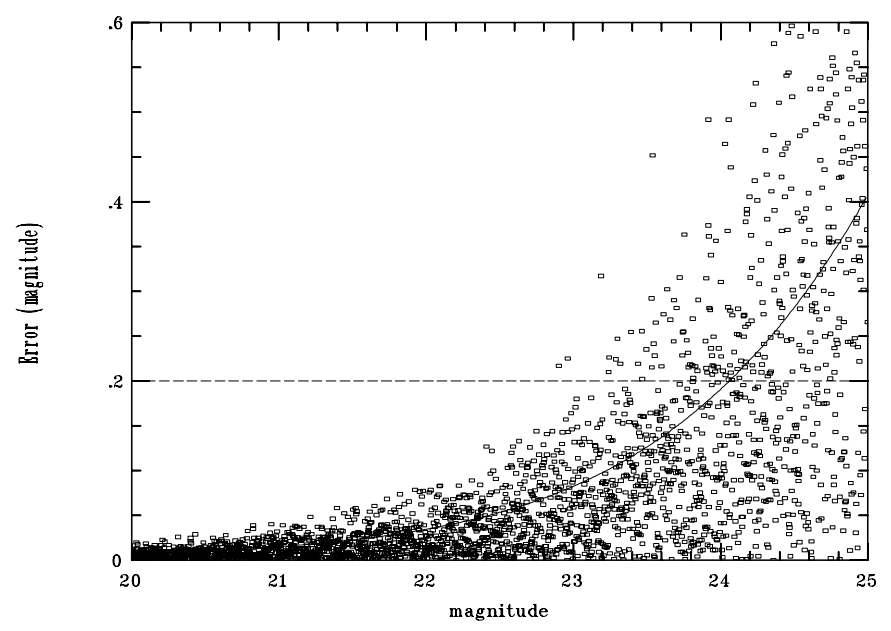

(b) Photometric errors.

Fig. 16. Expected astrometric and photometric errors for the ILMT, as a function of the $V$ stellar magnitude, corresponding to a PSF with a seeing of 1.0 arcsec, for a 1.000-trail through a $4 \times 4 C$-CCD. On each graph, the error curve and the chosen cut limit are shown.

simulations being independent of the star-trail position) and $N=56$ times those simulations with no TDI correction (i.e. 43008 error graphs).

Figure 18 illustrates the relative influence of the TDI mode on the limiting magnitude, for a seeing of 1.0 arcsec, using the PSF-fitting method, for the astrometry (top graph) and the photometry (bottom graph). Solid lines represent the variation of the limiting magnitude when no TDI corrector is set, while dashed lines represent the case when the TDI effects are perfectly corrected for. Note that because of the independence of the TDI corrected case with the star-trail position, the simulations are only made once for the four different considered 


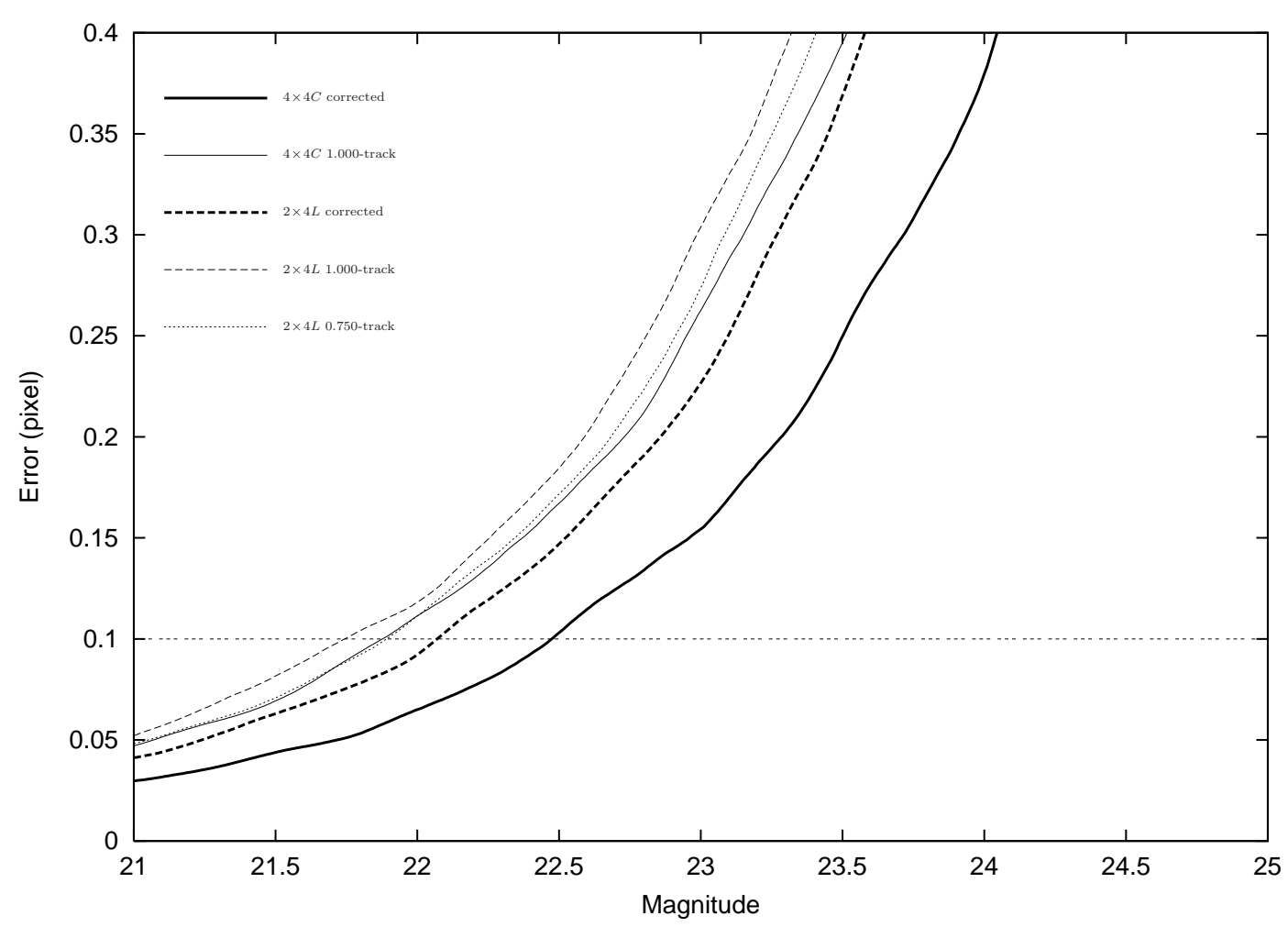

(a) Astrometry.

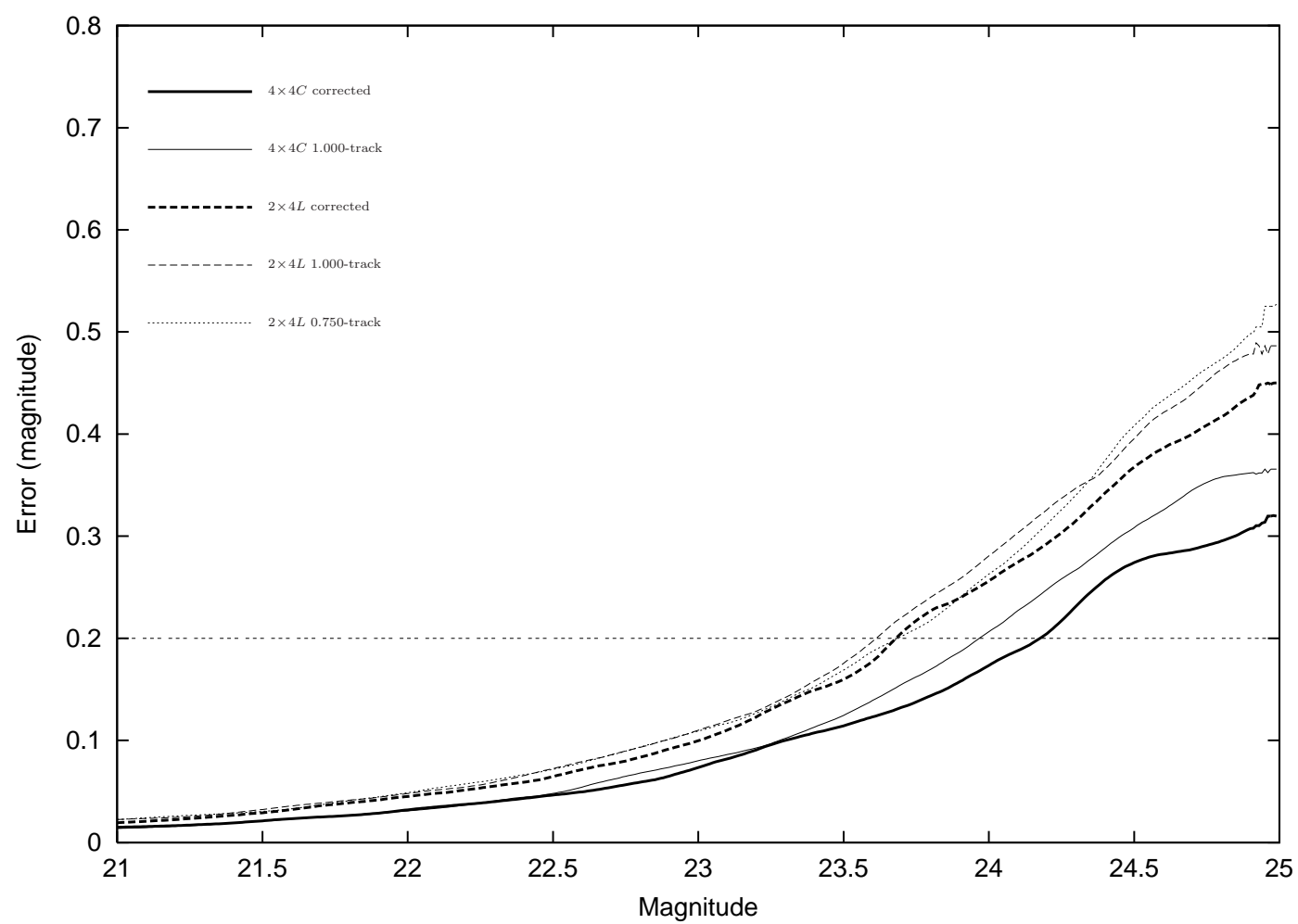

(b) Photometry.

Fig. 17. Comparison of the sliding median curves representing the error measurements, for different CCD mosaic configurations. All measurements were made with the PSF fitting method. The adopted seeing is 1.0 arcsec. 


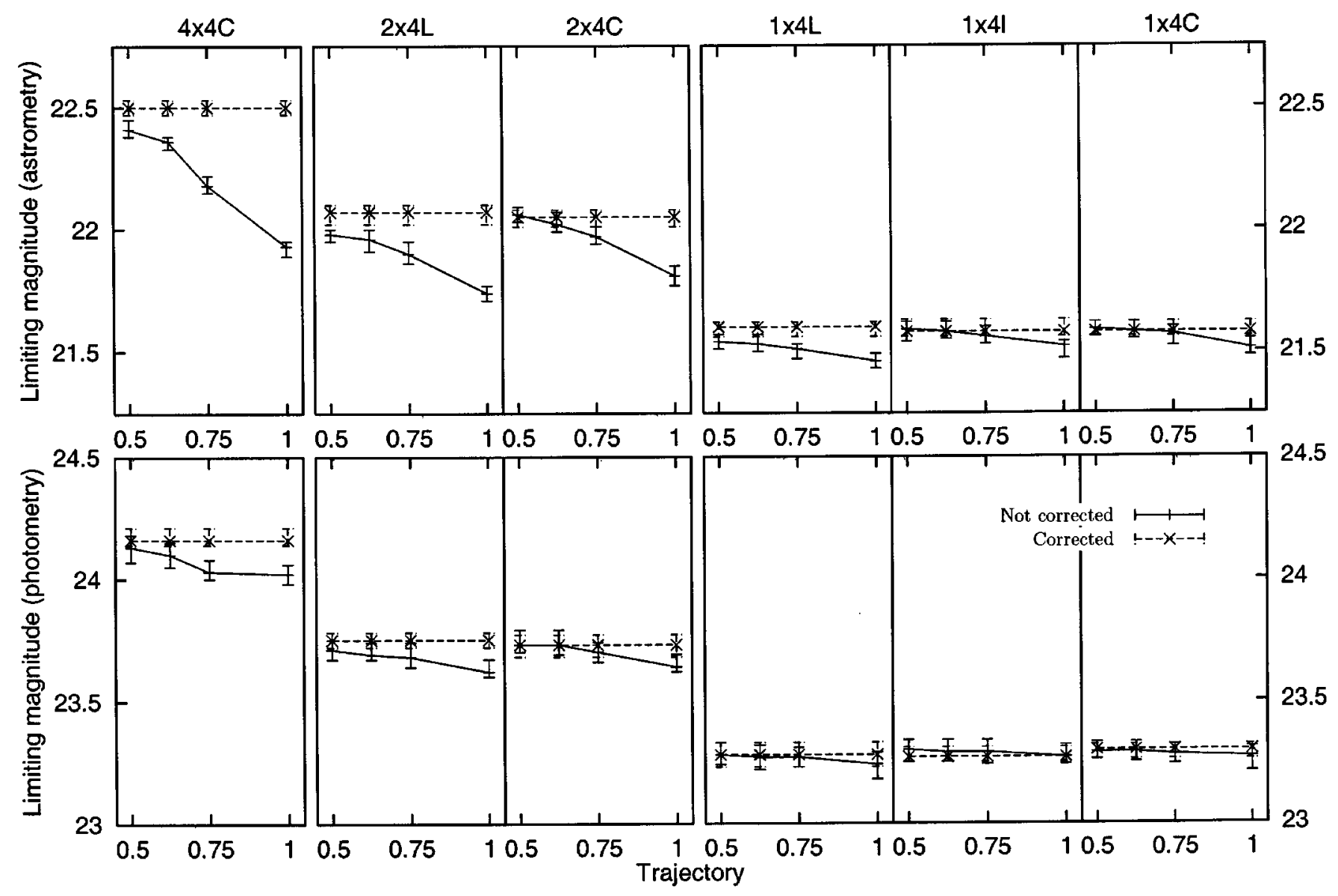

Fig. 18. Limiting magnitude for astrometry (top) and photometry (bottom), as a function of the star-trail position, for the various CCD positions defined in Fig. 1, and for a seeing of 1.0 arcsec. Solid lines represent the non corrected decrease in the limiting magnitude, while dashed lines represent the case where TDI is perfectly corrected for. For this example, the PSF-fitting method is used.

tracks. This is the reason why error bars are exactly constant for the dashed lines.

$\Delta m_{\text {lim }}$ represents the difference between these two - corrected and non corrected - values of the limiting magnitude. In fact, it represents the loss in field depth, when using the telescope without a TDI corrector. For a large $4 \times 4 C \mathrm{CCD}, \Delta m_{\text {lim }}$ is more critical when the star passes closer to the edges of the CCD: in astrometry for example, the loss is $-0.2 \mathrm{mag}$ for the 0.500 -track versus $-0.83 \mathrm{mag}$ for the 1.000 -track.

\subsection{Cutting out of the mosaic}

The photometric and astrometric effects resulting from cutting out the CCD mosaic along the East-West and North-South directions are discussed below.

\subsubsection{East-West cutting out}

This difference $\Delta m_{\text {lim }}$ becomes smaller when using narrower CCDs (along the East-West direction) (see $4 \times 4 C$ against $2 \times 4 C$ and $1 \times 4 C$ cases in Fig. 18). Note that for the same integration time, it is better to use a tilted CCD (for which the rows are aligned with the star trajectories), especially for the central stars (see $2 \times 4 L$ against $2 \times 4 C$, and $1 \times 4 L$ against $1 \times 4 C: \Delta m_{\lim }$ becomes nearly negligible).

Note that when dividing the CCD into a mosaic of twice narrower CCDs along the East-West direction, the integration time becomes of course twice shorter, and this decreases the limiting magnitude by a value of approximately $2.5 \log \sqrt{2}=0.376$. This is observed in our simulations, when the TDI effect is perfectly corrected for. The use of two narrow CCDs instead of a wider one leads to a brighter limiting magnitude, but this should in principle be balanced by correctly co-adding the two images (East and West). This is the reason why we pay much more attention to the relative value $\Delta m_{\lim }$ than to the absolute limiting magnitudes.

\subsubsection{North-South cutting out}

Using twice narrower CCDs along the North-South direction can also be useful. For example, the top graph in Fig. 18 shows that for a $4 \times 2 C$ CCD (0.750-track in a $4 \times 4 C$ single CCD), $\Delta m_{\text {lim }}$ is reduced to $-0.32 \mathrm{mag}$, instead of -0.57 . 
However, the small gaps between the CCDs cause a loss of data. This is a problem in declination and can be overcome with the CCD array slightly shifted along the North-South direction, between consecutive nights.

\subsubsection{Discussion}

From all these considerations, dividing the number of columns by two is a good choice (e.g. configurations 3 or 8 , from Fig. 3). Going to a configuration with eight or sixteen smaller CCDs would still be better but does not lead to a sufficient improvement to be justified.

Note that when comparing the limiting magnitudes for the aperture photometry and the PSF-fitting, we see an advantage of approximately $0.4 \mathrm{mag}$ in using the latter method when carrying out the photometric measurements. Of course, we took fixed parameters for a large number of stable simulations and it is likely that aperture photometry does suffer more from this assumption. But we can state that PSF-fitting is well suited to the TDI mode, because of the complex PSF when no corrector is set (see Sect. 4).

\section{The case of the Bordeaux astrometric camera}

In order to check our method, we have performed simulations corresponding to the observing and seeing conditions of another telescope used in the TDI mode.

The Bordeaux Observatory and the Instituto Astronomico e Geofisico of São Paulo use a CCD camera working in the drift-scan mode which is mounted on a meridian circle (Viateau et al. 1998). Astrometric and photometric measurements of a field around M35 have been carried out in the $V$ band.

The internal positional errors have been reported to be about \pm 0.04 arcsec for $9 \leq V \leq 14$, and \pm 0.15 arcsec for $V=15.5$. The internal accuracy of the magnitude results was better than about 0.05 mag for objects with $V \sim 12$ or 13 (Viateau et al. 1998).

\subsection{Star frame simulations}

For the case of the Bordeaux meridian circle, we have constructed a star frame filled with 2500 stars distributed in the magnitude range 8-16. We have measured their positions and magnitudes with the PSF-fitting method, and derived error estimates.

The $1024 \times 1024$ CCD camera used by Viateau et al. (1998) in Bordeaux (latitude $\ell=+44^{\circ} 50^{\prime}$ ) has a pixel size of $19 \mu \mathrm{m}$. The focal length of the meridian circle is $2.368 \mathrm{~m}$, corresponding to a scale of $1.6550 \mathrm{arcsec} / \mathrm{pixel}$. The telescope has an aperture of $0.20 \mathrm{~m}$. The declination of the M 35 star field is about $\delta=+24^{\circ}$.

In our simulations, a readout noise of 8.5 electrons is adopted. The value of the sky background is chosen to be 24 electrons/arcsec/sec, typical of a dark $V$ night sky brightness of $21.8 \mathrm{mag} / \mathrm{arcsec}$. Seeing is taken as 3.0 arcsec.
We calculate the limiting magnitude for an astrometric error of 0.024 pixel, i.e. 0.04 arcsec, and we take an upper limit of 0.05 mag for the photometric errors.

\subsection{Error measurements}

The results of our simulations and measurements are given in Table 1. There, we present the limiting magnitude for a star crossing the CCD without TDI effect (corrected) and for stars passing along the 0.500-trail, 0.700-trail and 0.950-trail, including the TDI effect (not corrected).

For the astrometric measurements, Table 1a shows the limiting magnitude to be $V \leq 14$, in good agreement with the results of Viateau et al. (1998). Considering the photometry, Table $1 \mathrm{~b}$ suggests that a better limiting magnitude could be obtained using the PSF-fitting method. But their scanning micrometer was primarily conceived as an astrometric device and the photometry was a by-product, mainly useful for identification purposes.

\section{Conclusions}

When deriving the uncertainties induced by the TDI mode on the photometric and astrometric measurements, and the feasibility of using the ILMT without a specific TDI corrector, we simulated images neglecting the usual aberrations (astigmatism, coma, distortion). But it is not only the question of using or not a TDI correction: we also have to measure its importance relative to the other aberrations. Here are some conclusions.

\subsection{Correction for aberrations and TDI}

The use of one large $4 \times 4$-CCD in single TDI mode, with a perfect TDI corrector (which is also supposed to perfectly correct the other aberrations) and with a seeing of $1.0 \mathrm{arcsec}$, leads to a limiting magnitude of $22.50_{-0.3}^{+0.3}$ in astrometry (for a maximum error of 0.04 arcsec), and of $24.16_{-0.2}^{+0.5}$ in photometry (for a maximum error of $0.2 \mathrm{mag}$ ).

\subsection{Correction for aberrations only}

For various reasons, we wondered whether it is possible to use the ILMT without any TDI corrector, and under which conditions.

In Sect. 3, we have shown that the star motion without such a corrector produces an elongation of the trace along the East-West and the South-North directions (respectively of $\left|E_{x}\right|=2.58$ arcsec and $\left|E_{y}\right|=0.65 \operatorname{arcsec}$ for the worst case at the considered declination, i.e. for a trajectory close to the southern edge). In order to obtain a PSF as circular as possible, i.e. an image with similar elongations in both directions, the CCD mosaic has to be composed of $4 \times 1$-CCDs (configuration 11 in Fig. 3).

In Sect. 4, we calculated that the TDI effects, for a seeing of 1.0 arcsec, widen the $F W H M$ of a star passing at the edge by $48.2 \%$. 
Table 1. The Bordeaux simulations with the PSF-fitting method, for the astrometry and the photometry. $m_{\lim [50]}, m_{\lim [16]}$ and $m_{\lim [84]}$ have been defined in Sect. 5.4.

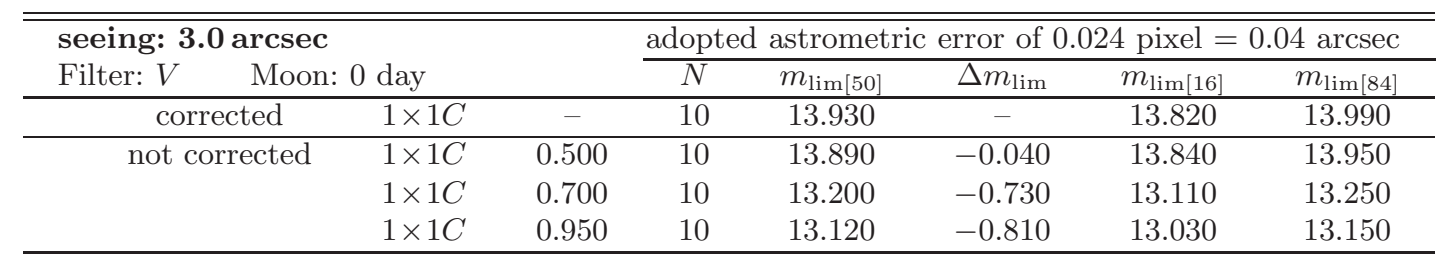

(a) Astrometry.

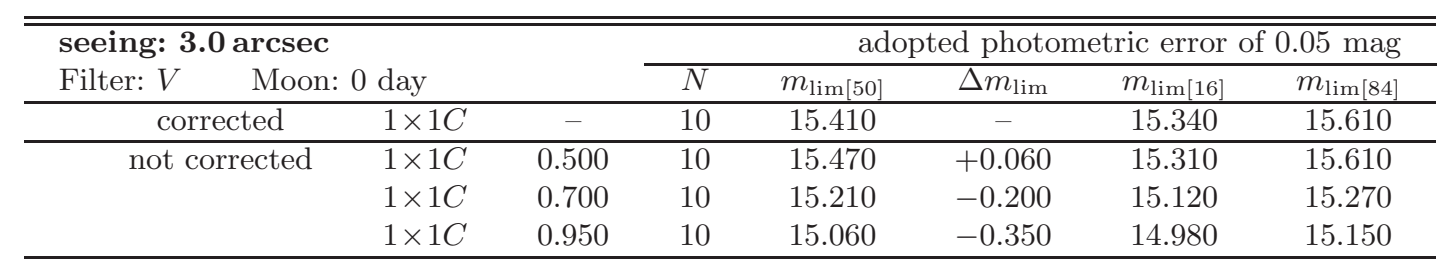

(b) Photometry.

Table 2. Synthesis of the comparison of the FWHM widening and loss in limiting magnitude, for each of the 15 proposed CCD mosaic configurations and an input seeing of 1.0 arcsec. For each configuration, the first row represents the configuration corresponding to Fig. 3. The second row indicates which single-CCD simulation and which track were used. The third row lists the FWHM widening. The fourth and fifth rows list the limiting magnitude loss (in magnitude, adopting a maximum error of 0.039 arcsec in astrometry and 0.2 mag in photometry).

\begin{tabular}{|c|c|c|c|c|c|}
\hline $\begin{array}{r}\text { configuration } \\
\text { single conf. } \\
F W H M \\
\text { astrometry } \\
\text { photometry }\end{array}$ & $\begin{array}{c}\text { Configuration } 1 \\
1 \times 4 \times 4 \\
4 \times 4 C \text { at } 1.000 \\
+0.479 \text { arcsec } \\
-0.57 \\
-0.14\end{array}$ & & & & \\
\hline $\begin{array}{r}\text { configuration } \\
\text { single conf. } \\
F W H M \\
\text { astrometry } \\
\text { photometry }\end{array}$ & $\begin{array}{c}\text { Configuration } 6 \\
2 \times 4 \times 2 \\
4 \times 4 C \text { at } 0.750 \\
+0.236 \text { arcsec } \\
-0.32 \\
-0.13\end{array}$ & $\begin{array}{c}\text { Configuration } 2 \\
2 \times 2 \times 4 \\
2 \times 4 L \text { at } 1.000 \\
+0.232 \text { arcsec } \\
-0.33 \\
-0.13\end{array}$ & $\begin{array}{c}\text { Configuration } 3 \\
2 \times 2 \times 4 \text { tilted } \\
2 \times 4 C \text { at } 1.000 \\
+0.191 \text { arcsec } \\
-0.24 \\
-0.09\end{array}$ & & \\
\hline $\begin{array}{r}\text { configuration } \\
\text { single conf. } \\
F W H M \\
\text { astrometry } \\
\text { photometry }\end{array}$ & 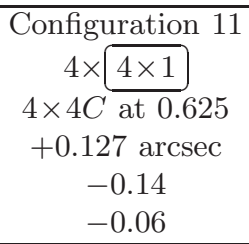 & 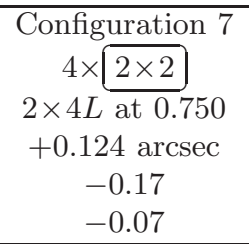 & $\begin{array}{l}\text { Configuration } 8 \\
4 \times 2 \times 2 \text { tilted } \\
2 \times 4 C \text { at } 0.750 \\
+0.070 \text { arcsec } \\
-0.08 \\
-0.03\end{array}$ & 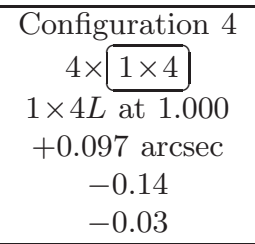 & $\begin{array}{c}\text { Configuration } 5 \\
\begin{array}{c}4 \times 1 \times 4 \\
1 \times 4 L \text { at } 1.000 \\
+0.065 \text { arcsec } \\
-0.07 \\
-0.04\end{array}\end{array}$ \\
\hline $\begin{array}{r}\text { configuration } \\
\text { single conf. } \\
F W H M \\
\text { astrometry } \\
\text { photometry }\end{array}$ & & $\begin{array}{c}\text { Configuration } 12 \\
\begin{array}{c}8 \times 2 \times 1 \\
2 \times 4 L \text { at } 0.625 \\
+0.093 \text { arcsec } \\
-0.11 \\
-0.06\end{array}\end{array}$ & $\begin{array}{c}\text { Configuration } 13 \\
8 \times 2 \times 1 \text { tilted } \\
2 \times 4 C \text { at } 0.625 \\
+0.040 \text { arcsec } \\
-0.03 \\
-0.00\end{array}$ & $\begin{array}{l}\text { Configuration } 9 \\
8 \times 1 \times 2 \\
\begin{array}{c}8 \times 4 L \text { at } 0.750 \\
+0.071 \text { arcsec } \\
-0.09 \\
-0.01\end{array}\end{array}$ & $\begin{array}{l}\text { Configuration } 10 \\
8 \times 1 \times 2 \text { tilted } \\
\begin{array}{c}1 \times 4 L \text { at } \\
0.750 \\
+0.036 \text { arcsec } \\
-0.01 \\
-0.02\end{array}\end{array}$ \\
\hline $\begin{array}{r}\text { configuration } \\
\text { single conf. } \\
F W H M \\
\text { astrometry } \\
\text { photometry }\end{array}$ & & & & $\begin{array}{c}\text { Configuration } 14 \\
16 \times 1 \times 1 \\
\begin{array}{c}1 \times 4 L \text { at } 0.625 \\
+0.063 \text { arcsec } \\
-0.07 \\
-0.01\end{array}\end{array}$ & 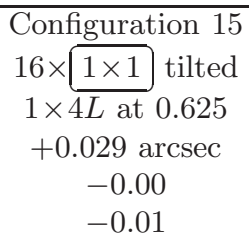 \\
\hline
\end{tabular}




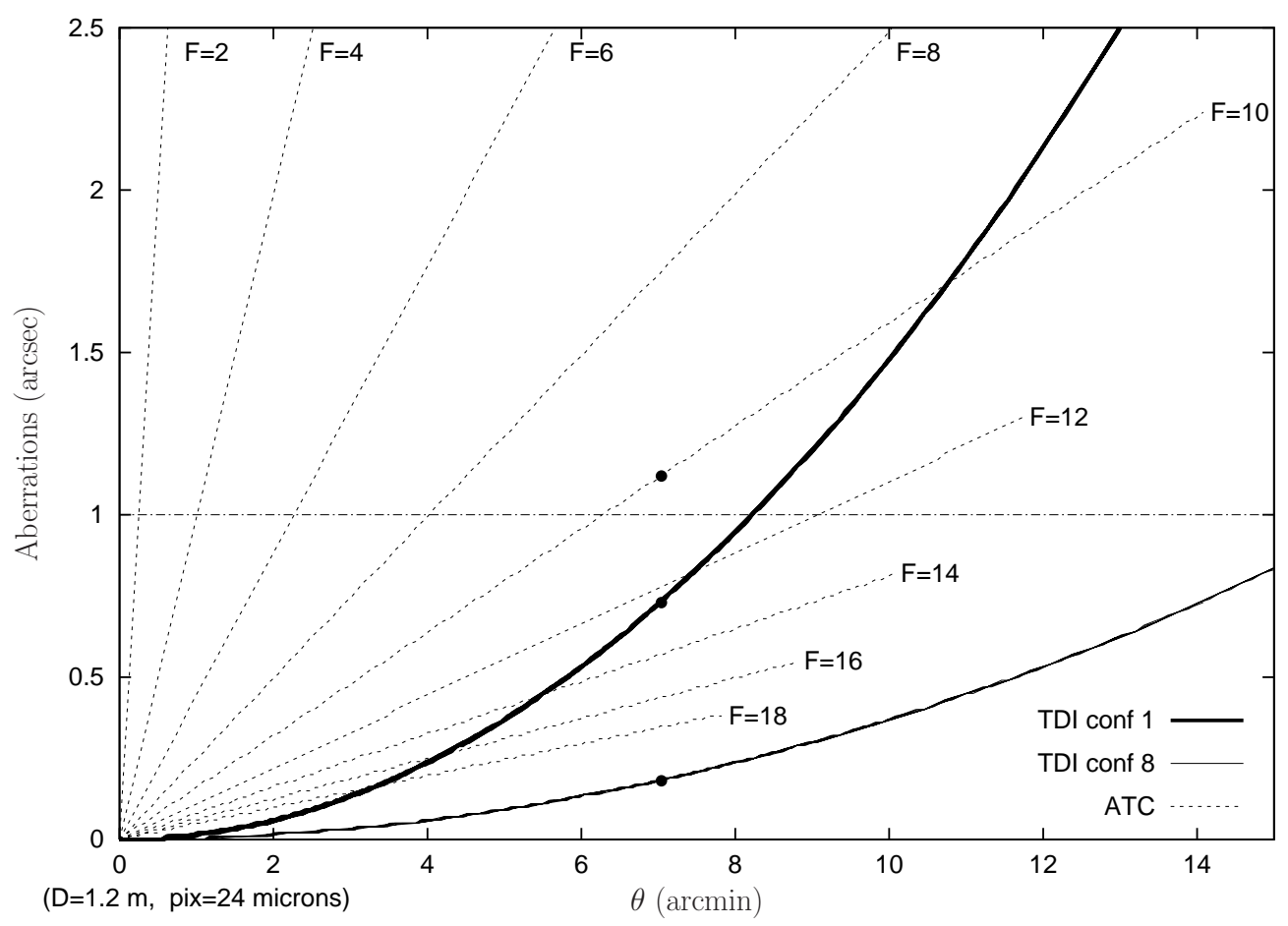

Fig. 19. $A T C$ for a telescope of $D=1.20 \mathrm{~m}$ and a camera with a pixel size of 24 microns (dashed lines) and the corresponding $\left|E_{x}\right|$ TDI elongation at the southern edge, for the ILMT latitude, for configuration 1 (upper solid line) and configuration 8 (lower solid line).

Analysis of the profile $F W H M$ and ellipticity (see Sect. 4), and of the limiting magnitude (see Sect. 5) shows some advantages in dividing the CCD by a factor two or four in both directions.

\subsection{Looking for the best solution}

Table 2 summarizes the main results of our analysis, where the configurations are sorted out according to the number of CCDs. For each of the mosaic configurations, the $F W H M$ widening and the loss in limiting magnitude (for astrometry and photometry) are given.

The configurations made of two CCDs (configurations 6,2 and 3 ) can lead to encouraging results but remain unsatisfactory: the $F W H M$ widening ranges between $19.2 \%$ and $23.7 \%$, the limiting magnitude loss between -0.24 and -0.33 in astrometry and between -0.09 and -0.13 in photometry. It seems better to divide the CCD along the East-West direction and tilt it to align the rows with the star trajectories (if possible): the best choice is configuration 3 .

The configurations made of four CCDs (configurations 11, 7, 8, 4 and 5) lead to much more satisfactory results. The FWHM increase ranges between $6.5 \%$ and $12.7 \%$, the limiting magnitude loss between -0.07 and -0.17 in astrometry and between -0.03 and -0.07 in photometry. Again, dividing the mosaic along the East-West direction (and tilting) significantly improves the results (configuration 8 and better 5). But these two latter also shorten the integration time and the limiting magnitude remains intrinsically better for configurations 11 (24.10).
Better results could be obtained by co-adding East and West images from configurations 8 (or 5).

The configurations made of more than four CCDs can in theory still improve the results, but the large number of CCDs involved then leads to other problems (cf. image co-addition, CCD camera construction, etc.)

\subsection{The ILMT project}

For the case of the ILMT, the best choice would probably be configuration 8 , taking into account that configuration 5 is practically more complex to construct, because there are three gaps instead of one along the star trajectory. Furthermore, these considerations disregard the difficulty of aligning the CCD rows with the star trajectories, giving then more consistency to configurations 4 and 7 .

Let us note that even with an absolutely perfect TDI corrector, which only corrects for the trajectory distortion, the discrete shifting takes place and there will be an inevitable effect in East-West widening.

\subsection{Without any correction}

For the case of a parabolic mirror, the distortion is null. But the angular sagital $(A S C)$ and tangential $(A T C)$ coma and the angular astigmatism $(A A S)$ are to be taken into account (e.g. Schroeder 1987):

$A S C=\frac{\theta}{16 F^{2}}$,
$A T C=3 A S C=\frac{3 \theta}{16 F^{2}}$, 
$A A S=\frac{\theta^{2}}{2 F}$

which are expressed in radians. $F=f / D$ is the focal ratio and $\theta$ is the angular distance to the axis (in radians).

For the ILMT project, these optical aberrations turn out to be much more important $(A T C=52$ arcsec and $A A S=1.5$ arcsec) than the image maximum elongations induced by the TDI $\left(\left|E_{x}\right|=2.58\right.$ arcsec and $\left|E_{y}\right|=0.65$ arcsec for the latitude of El Toco). This is why the ILMT project cannot work without a corrector. Note that the relative cost of the TDI correction in the global corrector budget is low.

But it is clear that a telescope with a smaller aperture and a camera with a more restricted field of view may be operated without any TDI corrector, by eventually using an adequate mosaic of narrower CCDs.

We may even wonder whether it is possible to run it without any aberration corrector, this issue being very interesting for those who want to get a very low cost survey telescope. Let us consider, for example, a mid-size telescope (e.g. $D=1.20 \mathrm{~m}$ ), with a not too large focal ratio in order to limit the dome height (e.g. $F=10$ ).

Let us compare the $A T C$, which is the most severe aberration (for low $\theta$, see Schroeder 1987), and the TDI East-West elongation $\left|E_{x}\right|$ which is always larger than $\left|E_{y}\right|$, for a square CCD at the edges. Figure 19 illustrates this comparison for a CCD whose pixel size is pix $=24 \mathrm{mi}-$ crons and for the latitude of the ILMT (El Toco).

Note that the angular TDI deformations are independent of the telescope characteristics such as $f$ and $D$ (see Eq. (1)), and consequently $F$. Here, for the comparison, they have been calculated at the edge, while the coma deformation has been evaluated at the corner (i.e. for $\sqrt{2} \cdot \theta$ ).
Indeed, the $A T C$ formula is avalaible for one impact, while we want to compare the deformations due to the whole integration.

Figure 19 clearly shows that it is possible to use such a telescope without any corrector, for a maximum aberration of about 1 arcsec. With a standard CCD camera (e.g. $2048 \times 2048$ pixels, i.e. $\theta=7.04 \mathrm{arcmin}$ ), $A T C$ would fall to $1.12 \operatorname{arcsec}(A A S$ to $0.09 \operatorname{arcsec})$, while the TDI elongations are $\left|E_{x}\right|=0.73$ arcsec and $\left|E_{y}\right|=0.18$ arcsec (see the black dots in Fig. 19). The TDI elongations are small but for higher latitudes, they will become more important. It is then useful to follow our previous considerations and to divide the camera into an adequate mosaic of CCDs. For example, the two lower black dots in Fig. 19 correspond to configurations 1 and 8.

\section{References}

Borra, E. F. 1982, J. R. Astron. Soc. Can., 76, 245

Gibson, B. K., \& Hickson, P. 1992, MNRAS, 258, 543

Hickson, P., \& Richardson, H. 1998, PASP, 110, 1081

Mailly, E. 1872, De l'Astronomie dans l'Académie Royale de Belgique, Rapport Seculaire, 1772-1872, 99

Poels, J., et al. 2001, Astronomical Data Analysis Software and Systems X, ASP Conf. Ser. (All the existing ILMT documentation may be found at the following URL: http://vela.astro.ulg.ac.be/lmt), 238, 97

Schroeder, D. 1987 (Astronomical Optics, Academic Press, San Diego), 95

Stone, R. C., Monet, D. G., Monet, A. K. B., et al. 1996, AJ, 111,1721

Viateau, B., et al. 1998, A\&AS, 134, 173 